LINGÜÍSTICA 



\title{
NOMBRES COMUNES Y TÉCNICOS DE LOS PECES DE AGUA DULCE DE COSTA RICA ${ }^{1}$
}

\author{
Arturo Angulo
}

\begin{abstract}
RESUMEN
Se presenta a continuación un listado que recopila 260 nombres comunes y 60 nombres técnicos utilizados en Costa Rica para denominar un total de 154 especies de peces de agua dulce residentes en el país (151 nativas y 3 exóticas). Se incluye información relativa al origen, significado y uso de tales nombres a escala regional, así como información actualizada referente al estatus nomenclatural y distribución geográfica de las especies tratadas. Esta información permitirá una comunicación más fluida entre técnicos, científicos, personas que toman decisiones y personas comunes que tienen fines de conservación.

Palabras clave: Ictiofauna dulceacuícola de Costa Rica, nomenclatura técnica y científica, nombres vernaculares, geografía lingüística, distribución.
\end{abstract}

\begin{abstract}
Set out below a list of 260 common names and 60 technical names used in Costa Rica, to name a total of 154 resident species of freshwater fishes (151 native and 3 exotic). Information about the origin, meaning and use of such names at regional level, as well as updated information concerning the nomenclatural status and geographic distribution of species is provided. This information will allow better communication between technicians, scientists, political decision takers and common people with conservation purposes.

Key words: Costa Rican freshwater fish fauna, technical and scientific nomenclature, vernacular names, linguistic geography, distribution.
\end{abstract}

\section{Introducción}

Diversas publicaciones, técnicas y científicas, destacando para Costa Rica los trabajos de Alpírez (1985), Bussing (1987, 1998), Bussing y López (1994, 2005, 2010) y Angulo et al. (2013), suelen agregar notas o listas en las que figuran, en compañía del nombre específico, algunos nombres técnicos o comunes de las especies tratadas. Tal propósito responde, entre otras razones, a la necesidad de coordinar el lenguaje científico con el popular para los efectos de facilitar, en la medida de lo posible, su difusión y acceso al conocimiento general (Martínez y Bonetto 1957: 3).

M.Sc. Arturo Angulo. Investigador Asociado, Sección de Ictiología, Museo de Zoología, Escuela de Biología, Universidad de Costa Rica.

Correo electrónico: arturo.angulosibaja@ucr.ac.cr / arturo.angs@gmail.com.

Recepción: 04- 03- 2013

Aceptación: 06- 05- 2013 
De acuerdo con lo expuesto por Martínez y Bonetto (1957: 3) y Mones y Kuhl (1991: 251), el hecho de coordinar ambos lenguajes (científico y popular), incluyendo en la medida de lo posible la variación nomenclatural existente a escala geográfica, puede ser provechoso desde distintas perspectivas. Un ejemplo de ello, en alusión a los objetivos del presente trabajo, es la vasta gama de conocimientos empíricos y observaciones valiosas de pescadores, ribereños y acuaritas, los cuales propiciarían, de cumplirse lo anterior, el desarrollo del conocimiento ictiológico, a nivel regional, en una relación más estrecha con el medio geográfico, permitiendo además encauzarlo hacia aspectos de significancia social y económica (Martínez y Bonetto 1957: 3).

El deseo comprensible de técnicos y científicos de producir listas de nombres comunes o técnicos únicos a nivel de especie, manifiesto en sus publicaciones, así como el de estadistas y comerciantes en sus transacciones, resulta, no obstante, contraproducente para los objetivos anteriormente expuestos, tal y como lo han discutido ya diversos autores (Martínez y Bonetto 1957: 3, Villeumier 1999: 71, Pardos 2001: 56, Chebez et al. 2010: 2). En este caso, la simplificación de nombres, conduce a una pérdida de tradiciones en el lenguaje local o popular y genera además, contrario a su propósito inicial, cierta confusión y problemas en la correcta identificación de las especies, de acuerdo a lo expuesto por Navas et al. (1991: 4) y Pardos (2001: 56), con repercusiones en los ámbitos ecológico, económico y social (Martínez y Bonetto 1957: 3, Navas et al. 1991: 7, Pardos 2001: 56, Chebez et al. 2010: 1-2).

Dado esto, se tiene entre manos una situación paradójica. Por un lado se tiene una gran diversidad de nombres comunes o vernaculares, reflejo del lenguaje vivo, que cambia y que es parte de la cultura humana; por el otro, existe la necesidad de una uniformidad nomenclatural que funcione como herramienta de comunicación entre las personas.

Procurar el estudio de la variación y el alcance de los nombres comunes de las especies para, de esta forma, conocer su valor y significación a escala local o regional, podría ser parte de la solución al problema planteado. Bajo esta premisa, cabe destacar, para Costa Rica, los trabajos de Fournier y García (1998) y León y Poveda (2000), en plantas; Mora (2000), en mamíferos; y Sandoval (2006), en aves; señalando además el vacío en el campo ictiológico pese la existencia de los trabajos ya citados de Alpirez (1985), Bussing $(1987,1998)$, Bussing y López $(1994,2005,2010)$ y Angulo et al. (2013).

Tomando esto en consideración, la presente investigación tuvo por objetivo determinar la variación, alcance, valor, uso y significancia local y regional de los nombres comunes y técnicos de algunas de las especies de peces de agua dulce de Costa Rica (las más comunes o de mayor importancia socio-económica), en vista de generar información que pueda resultar de utilidad a los fines anteriormente expuestos. Esto, aún cuando la lista resultante puede ser susceptible de ampliación o corrección en diferentes aspectos.

\section{Metodología}

Para el desarrollo de la presente investigación se tomaron como base los trabajos de Alpírez (1985), Bussing (1998), Bussing y López (1994, 2010) y Angulo et al. (2013), los cuales proporcionan listados no exhaustivos de nombres técnicos o comunes para la gran mayoría de las especies tratadas en este trabajo. Cabe destacar, que las especies incluidas en la presente revisión son aquellas que, con mayor frecuencia, se encuentran en los ambientes riverinos y lacustres del país, obviando, en muchos de los casos especies periféricas (principalmente marinas) asociadas a sistemas costero-estuarinos. 
En la presente, se incluyen numerosos datos/registros obtenidos de diversas fuentes (consulta directa a pescadores, ribereños, acuaristas e investigadores, de diversas localidades en todo el territorio costarricense, así como a través de la consulta de otras fuentes bibliográficas y bases de datos en línea (http//fishbase.org y http//fishwise.co.za)). Esta información fue compilada por el autor durante los últimos 5 años (2009-2013), en gran medida, gracias al apoyo y colaboración de múltiples personas y entidades, algunas de las cuales se mencionan en la sección de notas.

Para efectos del presente trabajo, se consideran nombres comunes o vernáculos aquellos de uso popular, en sentido estricto. Desde el punto de vista científico tales nombres no tienen porqué coincidir con una única especie; por el contrario, estos son, en la gran mayoría de los casos, nombres colectivos bajo los cuales se agrupan varias especies, géneros o familias, inclusive. Los nombres técnicos o vulgares, por su parte, corresponden a nombres artificiales creados por técnicos y científicos, asignados en correspondencia única a determinada especie. Tales nombres son generalmente asignados en alusión a una característica física, de comportamiento o ecológica de la especie, en referencia a su ámbito de distribución geográfico o en homenaje a distintas personalidades del ámbito científico o político; esto, con el objetivo de (tratar de) facilitar la comunicación con personas no familiarizadas con el lenguaje científico.

Con respecto a los nombres científicos se sigue a Angulo et al. (2013) y a Eschmeyer (2013) en la asignación del nombre válido, autor, año de descripción y familia de cada especie. La identidad de los especímenes, vivos o preservados, y fotografías, a partir de las cuales se obtuvo información relevante a los objetivos de este trabajo, fue corroborada en todos los casos mediante el uso de bibliografía especializada (Bussing 1998 y Bussing y López 1994, 2010) y la comparación con especímenes preservados depositados en la colección de peces del Museo de Zoología de la Universidad de Costa Rica (UCR). Finalmente, para facilitar el análisis de la información, se dividió el país en cinco regiones geográficas (Figura 1).

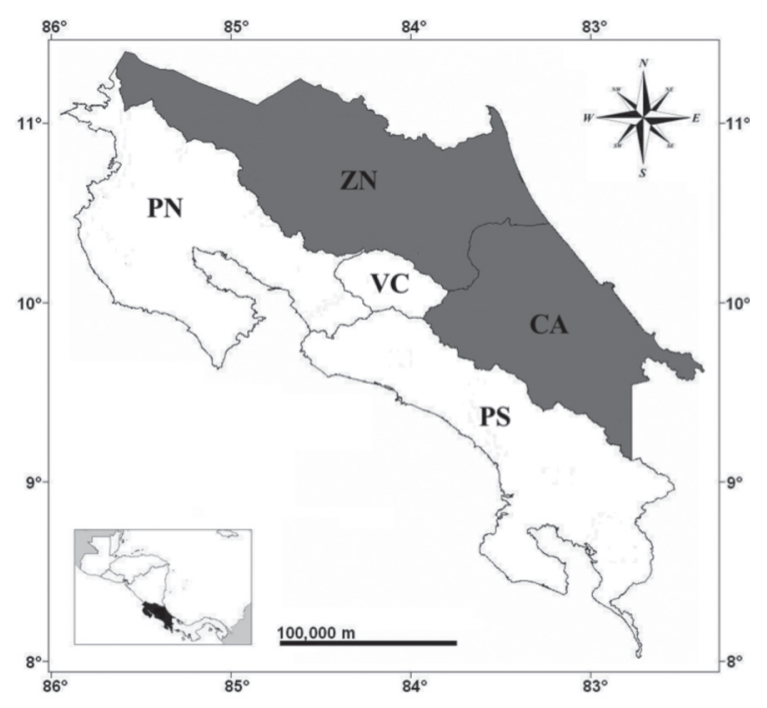

Figura 1. Regiones geográficas de Costa Rica: Pacífico Norte (PN), Pacífico Sur (PS), Zona Norte (ZN), Caribe (CA) y Valle Central (VC); atendiendo a los límites de las principales cuencas hidrográficas contenidas en cada región siguiendo a Bussing (1998: 16) y Angulo et al. (2013: 998) y considerando, además, razones históricas, socio-económicas y bio-geográficas. Las regiones sombreadas drenan al mar Caribe, las regiones sin sombrear drenan al océano Pacífico. 
A continuación, se presentan dos listados, alfabéticamente organizados, el primero de ellos presenta información taxonómica actualizada, datos de distribución y uso diferencial de los nombres comunes a nivel de especie y a nivel geográfico; el segundo, presenta información relativa al uso diferencial de los distintos nombres, sean comunes o técnicos, a nivel de taxa, así como el origen y significado de los mismos, cuando es conocido. La información contenida en ambos listados se complementa, por tanto se sugiere cruzar referencias para aprovechar de mejor forma la información presentada.

\section{Lista de las especies de peces de agua dulce de Costa Rica, distribución geográfica, nombres comunes y técnicos y uso regional}

Se presenta a continuación un listado taxonómico actualizado, alfabéticamente organizado, el cual incluye datos para un total de 154 especies de peces dulceacuícolas de Costa Rica. Los nombres científicos se presentan siguiendo a Angulo et al. (2013). Después del nombre científico se menciona el autor y el año de la descripción, posteriormente la familia a la cual pertenece la especie en cuestión, siguiendo a Angulo et al. (2013) y a Eschmeyer (2013). Cuando una determinada especie ha sido transferida a otro género que no es el original (acto nomenclatural denominado combinación) el nombre del autor de la descripción y el año se colocan entre paréntesis.

Los nombres comunes se presentan ordenados de acuerdo a su frecuencia de uso; de esta manera, para cada especie, el primer nombre en la lista representa el nombre cuyo uso es más extendido o más frecuentemente aplicado. Entre paréntesis se mencionan las diferentes regiones donde cada nombre es conocido o aplicado. Se subraya, la primera vez que se menciona, la(s) región(es) en la(s) cual(es) se tienen registros confirmados de la presencia de cada especie. Los datos de presencia/ausencia de cada especie según región (Figura 1) fueron obtenidos de la literatura (Bussing 1998, Bussing y López 1994, 2010, Angulo et al. 2013) y de la revisión de la base de datos de la colección ictiológica del Museo de Zoología de la UCR. Cabe mencionar que un determinado nombre común puede ser conocido en una región donde no necesariamente la especie a la cual se le denomina con ese nombre está presente, diversas son las razones que podrían explicar esta situación, la principal deriva del intercambio de información en la tradición popular.

Adicionalmente, se incluyen algunos nombres comunes utilizados por acuaristas, indicándose con el código (Ac), y nombres técnicos (Te), los cuales, en la mayoría de los casos, no se circunscriben a un lugar geográfico específico. Finalmente, todas las especies, con excepción de los casos señalados con un asterisco (*), son nativas de Costa Rica.

Achirus mazatlanus (Steindachner, 1869); Achiridae: Lenguado (N, $\underline{\mathrm{PS}}, \mathrm{ZN}, \mathrm{CA}, \mathrm{VC})$, Lenguado redondo (PN, PS), Pez hoja (PN, PS, CA, VC), Suela (PN, PS), Suela arepita (PN, PS), Suela de Mazatlan (Te).

Agonostomus monticola (Bancroft, 1834); Mugilidae: Tepemechín (PN, $\underline{\mathrm{PS}}, \underline{\mathrm{ZN}}, \underline{\mathrm{CA}}$, VC), Mechín (PN, PS, ZN, CA), Machín (ZN, VC), Lisa (PN, PS, CA, VC), Lisa de río (PN, PS, CA), Lisa de montaña (PS, ZN, CA), Sabalete montañero (PN, PS).

Alfaro cultratus (Regan, 1908); Poeciliidae: Olomina (N, $\underline{\mathrm{PS}} \underline{\mathrm{ZN}}, \underline{\mathrm{CA}}, \mathrm{VC}, \mathrm{Ac})$, Alumina (PN, PS, VC, Ac), Pepesca (PN, ZN), Olomina de Alfaro (Te).

Amatitlania kanna Schmitter-Soto, 2007; Cichlidae: Mojarra (PN, PS, ZN, CA, VC), Mojarra rayada (PN, ZN, CA, VC), Convicto (ZN, CA, VC, Ac), Mojarrita (PN, PS, 
ZN, CA), Chogorra (ZN, CA), Picaculo (ZN), Congo (PN), Burra (PN, ZN, CA), Carate $(\mathrm{ZN})$, Sargento $(\mathrm{ZN})$.

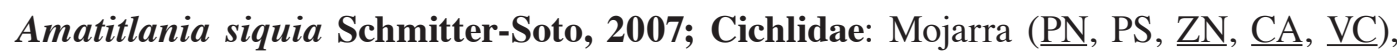
Mojarra rayada (PN, ZN, CA, VC), Convicto (ZN, CA, VC, Ac), Mojarrita (PN, PS, $\mathrm{ZN}, \mathrm{CA})$, Chogorra (ZN, CA), Picaculo (ZN), Congo (PN), Burra (PN, ZN, CA), Carate (ZN), Sargento (ZN).

Amphilophus alfari (Meek, 1907); Cichlidae: Mojarra (Nㅗ, PS, $\underline{\mathrm{ZN}}, \underline{\mathrm{CA}}, \underline{\mathrm{VC}})$, Mojarra pastel (VC, Ac), Mojarra de Alfaro (Te).

Amphilophus altifrons (Kner, 1863); Cichlidae: Mojarra (PN, PS, ZN, CA, VC).

Amphilophus bussingi Loiselle, 1997; Cichlidae: Mojarra (PN, PS, ZN, CA, VC), Mojarra real (CA, VC, Ac), Mojarra de Bussing (Ac, Te).

Amphilophus citrinellus (Günther, 1864); Cichlidae: Mojarra (PN, PS, ZN, $\underline{\mathrm{CA}}$, VC), Mojarra Midas (ZN, VC, Ac), Cíclido Midas (ZN, VC, Ac).

Amphilophus diquis (Bussing, 1974); Cichlidae: Mojarra (PN, PS, ZN, CA, VC), Mojarra del Diquís (Te).

Amphilophus longimanus (Günther, 1867); Cichlidae: Mojarra (PN, PS, $\underline{\mathrm{ZN}}, \mathrm{CA}, \mathrm{VC})$, Mojarra de pecho rojo (ZN, Ac), Pecho rojo (ZN), Carate (ZN), Cholesca (ZN).

Amphilophus lyonsi (Gosse, 1966); Cichlidae: Mojarra (PN, PS, ZN, CA, VC).

Amphilophus rhytisma (López S., 1983); Cichlidae: Mojarra (PN, PS, ZN, CA, VC), Mojarra perla (CA, VC, Ac), Mojarra real (CA, VC, Ac).

Amphilophus rostratus (Gill, 1877); Cichlidae: Mojarra (PN, PS, ZN, CA, VC), Masamiche $(\mathrm{ZN})$, Mojarra dorada (ZN), Mojarra amarilla (ZN), Mojarr picuda (ZN), Carate $(\mathrm{ZN})$.

Andinoacara coeruleopunctatus (Kner, 1863); Cichlidae: Mojarra (PN, PS, ZN, CA, VC), Mojarrita (PN, PS, ZN, CA), Chogorra (PS).

Anguilla rostrata (Lesueur, 1817); Angullidae: Anguila (PN, PS, $\underline{\mathrm{ZN}}, \underline{\mathrm{CA}}, \mathrm{VC}, \mathrm{Ac})$, Anguila de mar (ZN, CA), Anguila verdadera (ZN, CA), Anguila Americana (Te).

Archocentrus centrarchus (Gill, 1877); Cichlidae: Mojarra (PN, PS, $\underline{\mathrm{ZN}}, \underline{\mathrm{CA}}, \mathrm{VC})$, Mojarra rayada $(\mathrm{ZN})$, Tilapia $(\mathrm{ZN})$.

Archocentrus multispinosus (Günther, 1867); Cichlidae: Mojarra (PN, PS, ZN, CA, VC), Mojarra arcoiris (ZN, Ac), Cholesca (ZN), Tilapia (ZN).

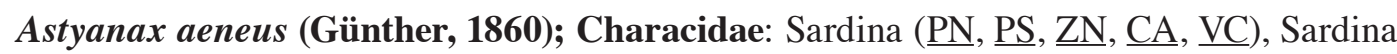
de río (PN, PS, ZN, CA, VC), Sardinilla (PN, PS, ZN, CA), Sabalete (ZN, CA), Sábalo (ZN), Sardina común (PN, PS, ZN, CA), Plateada (PN, PS, ZN, CA), Machaca (ZN, CA), Coliroja (PS, ZN, CA), Picaculo (PN, ZN), Golosa (PS), Tetra (VC, Ac), Mojarra (PN, PS, CA).

Astyanax cocibolca Bussing, 2008; Characidae: Sardina (PN, PS, ZN, CA, VC), Sardina lagunera (ZN), Sardinilla (PN, PS, ZN, CA), Sabalete (ZN, CA), Sardinita (PN, PS, ZN, CA), Plateada (PN, PS, ZN, CA), Tetra (VC, Ac), Sardina del lago Cocibolca (Te). 
Astyanax orthodus Eigenmann, 1907; Characidae: Sardina (PN, PS, ZN, CA, VC), Sardina blanca (CA), Tetra (VC, Ac).

Atherinella argentea Chernoff, 1986; Atherinopsidae: Sardina (N, $\underline{\mathrm{PS}}, \mathrm{ZN}, \mathrm{CA}, \mathrm{VC})$, Sardina plateada (PN, PS, ZN, CA), Pejerrey (PN, PS, ZN, CA), Sardinilla (PN, PS, ZN, CA), Plateadita (PN, PS, ZN, CA), Plateada (PN, PS, ZN, CA), Alfiler (PN, PS, $\mathrm{ZN}, \mathrm{CA})$.

Atherinella chagresi (Meek y Hildebrand, 1914); Atherinopsidae: Sardina (PN, PS, ZN, $\underline{\mathrm{CA}}$, VC), Sardina plateada (PN, PS, ZN, CA), Pejerrey (PN, PS, ZN, CA), Sardinilla (PN, PS, ZN, CA), Plateadita (PN, PS, ZN, CA), Plateada (PN, PS, ZN, CA), Alfiler (PN, PS, ZN, CA), Pejerrey del Chagres (Te).

Atherinella hubbsi (Bussing, 1979); Atherinopsidae: Sardina (PN, PS, ZN, CA, VC), Sardina plateada (PN, PS, ZN, CA), Pejerrey (PN, PS, ZN, CA), Sardinilla (PN, PS, ZN, CA), Plateadita (PN, PS, ZN, CA), Plateada (PN, PS, ZN, CA), Alfiler (PN, PS, $\mathrm{ZN}, \mathrm{CA})$, Pejerrey de Hubbs (Te).

Atherinella milleri (Bussing, 1979); Atherinopsidae: Sardina (PN, PS, ZN, CA, VC), Sardina plateada (PN, PS, ZN, CA), Pejerrey (PN, PS, ZN, CA), Sardinilla (PN, PS, ZN, CA), Plateadita (PN, PS, ZN, CA), Plateada (PN, PS, ZN, CA), Alfiler (PN, PS, $\mathrm{ZN}, \mathrm{CA})$, Pejerrey de Miller (Te).

Atherinella sardina (Meek, 1907); Atherinopsidae: Sardina (PN, PS, ZN, CA, VC), Sardina plateada (PN, PS, ZN, CA), Pejerrey (PN, PS, ZN, CA), Sardinilla (PN, PS, ZN, CA), Plateadita (PN, PS, ZN, CA), Plateada (PN, PS, ZN, CA), Alfiler (PN, PS, $\mathrm{ZN}, \mathrm{CA})$.

Atractosteus tropicus Gill, 1863; Lepisosteidae: Gaspar (PN, PS, $\underline{\mathrm{ZN}}, \underline{\mathrm{CA}}, \mathrm{VC}, \mathrm{Ac})$, Pejelagarto (ZN, VC), Gaspar tropical (Te).

Awaous banana (Valenciennes, 1837); Gobiidae: Chupapiedras ( $\underline{\mathrm{PN}}, \mathrm{PS}, \underline{\mathrm{ZN}}, \underline{\mathrm{CA}}, \mathrm{VC}$, Ac), Lamearenas (PN, PS, ZN, CA), Gobio (PN, PS, ZN, CA, VC, Ac), Gobio de río (PN, PS, ZN, CA, VC, Ac), Banana (ZN, CA), Guab(v)ina (PN, PS, ZN, CA).

Awaous transandeanus (Günther, 1861); Gobiidae: Chupapiedras (N, $\underline{P S}, \mathrm{ZN}, \mathrm{CA}, \mathrm{VC}$, Ac), Lamearenas (PN, PS, ZN, CA), Gobio (PN, PS, ZN, CA, VC, Ac), Gobio de río (PN, PS, ZN, CA, VC, Ac), Guab(v)ina (PN, PS, ZN, CA).

Belonesox belizanus Kner, 1860; Pociliidae: Gasparcillo ( $\underline{\mathrm{ZN}}, \underline{\mathrm{CA}})$, Pepesca gaspar (ZN), Olomina picuda (ZN, CA), Picuda (ZN, CA), Alumina picuda (ZN, CA), Pepesca (ZN)

Brachyhypopomus occidentalis (Regan, 1914); Hypopomidae: (Pez) Cuchillo (PN, ZN, $\underline{\mathrm{CA}}, \mathrm{Ac}),(\mathrm{Pez})$ Navaja (PN, ZN, CA), (Pez) Machete (PN, ZN, CA), Anguila (PN, PS, ZN, CA, VC), Anguila falsa (PN, PS, ZN, CA), Madre de barbudo (ZN, CA), (Pez) Cuchillo aplanado (Te).

Brachyrhaphis cascajalensis (Meek y Hildebrand, 1913); Poeciliidae: Olomina (PN, PS, $\mathrm{ZN}, \underline{\mathrm{CA}}, \mathrm{VC}, \mathrm{Ac})$, Alumina (PN, PS, ZN, CA, VC), Pepesca (PN, ZN), Olomina del Cascajal (Te).

Brachyrhaphis holdridgei Bussing, 1967; Poeciliidae: Olomina (PN, PS, ZN, CA, VC, Ac), Alumina (PN, PS, ZN, CA, VC), Pepesca (PN, ZN), Olomina de Holdridge (Te). 
Brachyrhaphis olomina (Meek, 1914); Poeciliidae: Olomina (PN, PS, ZN, CA, VC, Ac), Alumina (PN, PS, ZN, CA, VC), Pepesca (PN, ZN).

Brachyrhaphis parismina (Meek, 1912); Poeciliidae: Olomina (PN, PS, ZN, $\underline{\mathrm{CA}}$, VC, Ac), Alumina (PN, PS, ZN, CA, VC), Pepesca (PN, ZN), Olomina del Parismina (Te).

Brachyrhaphis roseni Bussing, 1988; Poeciliidae: Olomina (PN, $\underline{\mathrm{PS}}, \mathrm{ZN}, \mathrm{CA}, \mathrm{VC}, \mathrm{Ac})$, Alumina (PN, PS, ZN, CA, VC), Pepesca (PN, ZN), Olomina de Rossen (Te).

Brachyrhaphis terrabensis (Regan, 1907); Poeciliidae: Olomina (PN, PS, ZN, CA, VC, Ac), Alumina (PN, PS, ZN, CA, VC), Pepesca (PN, ZN), Olomina del Térraba (Te).

Bramocharax bransfordii Gill, 1877; Characidae: Sardina (PN, PS, ZN, CA, VC), Sardina picuda (ZN, CA), Picuda (ZN), Sábalo (ZN), Sabalete (ZN, CA), Sardina hocicuda (ZN, CA), Sardina dientona (ZN).

Brycon behreae Hildebrand, 1938; Characidae: Machaca (PN, PS, ZN, CA, VC), Sábalo (PN, ZN, CA), Sabalete (ZN, CA), Machaca del Pacífico (Te).

Brycon costaricensis Angulo y Gracian-Negrete, 2013; Characidae: Machaca (PN, PS, $\underline{\mathrm{ZN}}, \underline{\mathrm{CA}}, \mathrm{VC})$, Sábalo (PN, ZN, CA), Sabalete (ZN, CA), Machaca del Atlántico (Te).

Caranx caballus Günther, 1868; Carangidae: Jurel (PN, PS, ZN, CA, VC), Caballa (PN, PS, ZN, CA), Bonito (PN, PS, ZN, CA), Cocinero (PN), Chumbo (PN), Cojinua (PS), Cojinoa (PS), Cojinua chata (PS), Jurel ojón (PS), Jurel verde (Te).

Caranx caninus Günther, 1867; Carangidae: Jurel (N, $\underline{\mathrm{PS}}, \mathrm{ZN}, \mathrm{CA}, \mathrm{VC})$, Jurel toro (PN, PS), Caballa (PN, PS, ZN, CA), Bonito (PN, PS, ZN, CA), Sargentillo (PN, PS), Jurel del Pacífico.

Caranx latus Agassiz, 1831; Carangidae: Jurel (PN, PS, $\underline{\mathrm{ZN}}, \underline{\mathrm{CA}}, \mathrm{VC})$, Jurel cola amarilla/ de cola amarilla (PN, PS), Jurel blanco (ZN, CA), Bonito (PN, PS, ZN, CA), Jurel ojón (ZN, CA), Caballa (PN, PS, ZN, CA), Jack (ZN, CA, VC).

Carcharhinus leucas (Müller y Henle, 1839); Carcharhinidae: Tiburón toro (PN, $\underline{P S}$, $\underline{\mathrm{ZN}}, \mathrm{CA}, \mathrm{VC})$, Madrecazón (PS, ZN).

Carlana eigenmanni (Meek, 1912); Characidae: Sardina (PN, PS, ZN, $\underline{\mathrm{CA}}, \mathrm{VC})$, Sardinita (PN, PS, ZN, CA, VC), Tetra (Co,VC, Ac).

Cathorops steindachneri (Gilbert y Starks, 1904); Ariidae: Bagre (N, PS, ZN, CA, VC), Cuminate (PN, PS, ZN, CA), Bagre baboso (PN, PS), Cuminate baboso (PN, PS), Barbudo (PN, PS, ZN, CA, VC), Chimbembe (PN, PS), Pez gato (Ac), Bagre de Steindachner $(\mathrm{Te})$.

Cathorops tuyra (Meek y Hildebrand, 1923); Ariidae: Bagre (PN, PS, ZN, CA, VC), Cuminate (PN, PS, ZN, CA), Bagre besucón (PN, PS), Cuminate besucón (PN, PS), Barbudo (PN, PS, ZN, CA, VC), Chimbembe (PN, PS), Pez gato (Ac).

Centropomus medius Günther, 1864; Centropomidae: Róbalo (N, $\underline{\mathrm{PS}}, \mathrm{ZN}, \mathrm{CA}, \mathrm{VC})$, Robalo (PN, PS, ZN, CA, VC), Róbalo/Robalo aleta manchada (PN, PS), Róbalo/ Robalo ojón (PN, PS), Guajale (PN, PS), Gualaje aleta manchada (PN, PS).

Centropomus nigrescens Günther, 1864; Centropomidae: Róbalo (N, $\underline{\mathrm{PS}}, \mathrm{ZN}, \mathrm{CA}$, VC), Robalo (PN, PS, ZN, CA, VC), Róbalo/Robalo negro (PN, PS), Róbalo/Robalo redondo $(\mathrm{PN})$. 
Centropomus parallelus Poey, 1860; Centropomidae: Calva ( $\underline{\mathrm{ZN}}, \underline{\mathrm{CA}})$, Róbalo (PN, PS, ZN, CA, VC), Robalo (PN, PS, ZN, CA, VC), Róbalo/Robalo de escama pequeña (ZN, CA).

Centropomus pectinatus Poey, 1860; Centropomidae: Róbalo (PN, PS, $\underline{\mathrm{ZN}}, \underline{\mathrm{CA}}, \mathrm{VC})$, Robalo (PN, PS, ZN, CA, VC), Tarpón (ZN, CA), Róbalo/Robalo constantino (ZN, CA), Gualaje del Atlántico (Te).

Centropomus robalito Jordan y Gilbert, 1882; Centropomidae: Róbalo ( $\underline{\mathrm{PN}}, \underline{\mathrm{PS}}, \mathrm{ZN}$, CA, VC), Robalo (PN, PS, ZN, CA, VC), Robalito (PN, PS), Róbalo/Robalo de aleta amarilla (PN, PS).

Centropomus undecimalis (Bloch, 1792); Centropomidae: Róbalo (PN, PS, $\underline{\mathrm{ZN}}, \underline{\mathrm{CA}}, \mathrm{VC})$, Robalo (PN, PS, ZN, CA, VC), Róbalo/Robalo blanco (ZN, CA).

Centropomus viridis Lockington, 1877; Centropomidae: Róbalo (N, $\underline{\mathrm{PS}}, \mathrm{ZN}, \mathrm{CA}, \mathrm{VC})$, Robalo (PN, PS, ZN, CA, VC), Róbalo/Robalo plateado (PN, PS).

Citharichthys spilopterus Günther, 1862; Paralichthyidae: Lenguado (PN, PS, ZN, $\underline{\mathrm{CA}}$, VC), (Pez) Hoja (PN, PS, CA, VC), Pez plano (PN, PS, ZN, CA, VC).

Citharichthys uhleri Jordan, 1889; Paralichthyidae: Lenguado (PN, PS, $\underline{\mathrm{ZN}}, \underline{\mathrm{CA}}, \mathrm{VC})$, (Pez) Hoja (PN, PS, CA, VC), Pez plano (PN, PS, ZN, CA, VC).

Cryptoheros myrnae (Loiselle, 1997); Cichlidae: Mojarra (PN, PS, ZN, CA, VC), Chogorra (CA), Mojarra topaz (CA, Ac), Mojarra de Myrna (Te).

Cryptoheros sajica (Bussing, 1974); Cichlidae: Mojarra (PN, PS, ZN, CA, VC), Chogorra (PS).

Cryptoheros septemfasciatus (Regan, 1908); Cichlidae: Mojarra (PN, PS, ZN, $\underline{\mathrm{CA}}$, VC).

Cynodonichthys fuscolineatus (Bussing, 1980); Rivulidae: Olomina (N, PS, ZN, CA, VC), Alumina (PN, PS, ZN, CA, VC), Killi (VC, Ac), Saltón (PN, PS, ZN, CA, VC), Olomina del Arenal (Te).

Cynodonichthys glaucus (Bussing, 1980); Rivulidae: Olomina (PN, PS, ZN, CA, VC), Alumina (PN, PS, ZN, CA, VC), Killi (VC, Ac), Saltón (PN, PS, ZN, CA, VC), Olomina azul (Te).

Cynodonichthys hildebrandi (Myers, 1927); Rivulidae: Olomina (PN, PS, ZN, CA, VC), Alumina (PN, PS, ZN, CA, VC), Killi (VC, Ac), Saltón (PN, PS, ZN, CA, VC), Olomina moteada (Te), Olomina de Hildebrand (Te).

Cynodonichthys isthmensis (Garman, 1985); Rivulidae: Olomina (N, PS, ZN, $\underline{\mathrm{CA}}, \underline{\mathrm{VC}}$ ), Alumina (PN, PS, ZN, CA, VC), Killi (VC, Ac), Saltón (PN, PS, ZN, CA, VC).

Cynodonichthys rubripunctatus (Bussing, 1980); Rivulidae: Olomina (PN, PS, ZN, CA, VC), Alumina (PN, PS, ZN, CA, VC), Killi (VC, Ac), Saltón (PN, PS, ZN, CA, VC).

Cynodonichthys siegfriedi (Bussing, 1980); Rivulidae: Olomina (PN, $\underline{\mathrm{PS}}, \mathrm{ZN}, \mathrm{CA}$, VC), Alumina (PN, PS, ZN, CA, VC), Killi (VC, Ac), Saltón (PN, PS, ZN, CA, VC), Olomina moteada (Te), Olomina de Siegfred (Te).

Cynodonichthys uroflammeus (Bussing, 1980); Rivulidae: Olomina (PN, PS, ZN, CA, VC), Alumina (PN, PS, ZN, CA, VC), Killi (VC, Ac), Saltón (PN, PS, ZN, CA, VC), Olomina flama (Te). 
Cyphocharax magdalenae (Steindachner, 1879); Curimatidae: Sardina (PN, PS, ZN, CA, VC), Capani (PS), Capaniz (PS), Carpa (PN, PS, ZN, CA, VC), Plateada (PS), Plateadita (PS).

Diapterus rhombeus (Cuvier, 1829); Gerreidae: Palmito ( $\underline{\mathrm{ZN}}, \underline{\mathrm{CA}}$ ), Mojarra (PN, PS, ZN, CA, VC), Mojarra de estero (ZN, CA), Mojarra plateada (PN, PS, ZN, CA), Mojarra caitipia (ZN, CA).

Dormitator latifrons (Richardson, 1844); Eleotridae: Pocoyo ( $\underline{\mathrm{PN}}, \underline{\mathrm{PS}})$, Guab(v)ina (PN, PS, ZN, CA, VC), Pez perro (PN, PS, ZN, CA), Guarasapa (CA), Dormilón (PN, PS, ZN, CA), Pocoyo del Pacífico (Te).

Dormitator maculatus (Bloch, 1792); Eleotridae: Guab(v)ina (PN, PS, $\underline{\mathrm{ZN}}, \underline{\mathrm{CA}}, \mathrm{VC})$, Pipón (ZN, CA), Guarasapa (CA), Pocoyo (PN, PS), Pez perro (PN, PS, ZN, CA), Dormilón (PN, PS, ZN, CA), Pocoyo del Atlántico (Te).

Dorosoma chavesi Meek, 1907; Clupeidae: Sardina (PN, PS, ZN, CA, VC), Sardinilla (PN, PS, ZN, CA, VC), Sabalete (ZN), Sardina de río (PN, PS, ZN, CA).

Eleotris amblyopsis (Cope, 1871); Eleotridae: Guab(v)ina (PN, PS, ZN, $\underline{\mathrm{CA}}, \mathrm{VC}), \mathrm{Pez}$ perro (PN, PS, ZN, CA), Lucia (CA), Dormilón (PN, PS, ZN, CA).

Eleotris picta Kner, 1863; Eleotridae: Guab(v)ina (N, $\underline{\mathrm{PS}}, \mathrm{ZN}, \mathrm{CA}, \mathrm{VC})$, Vieja (N, $\underline{\mathrm{PS}})$, Pez perro (PN, PS, ZN, CA), Dormilón (PN, PS, ZN, CA), Dormilón manchado (Te).

Eleotris pisonis (Gmelin, 1789); Eleotridae: Guab(v)ina (PN, PS, $\underline{\mathrm{ZN}}, \underline{\mathrm{CA}}$, VC), Pez perro (PN, PS, ZN, CA), Dormilón (PN, PS, ZN, CA).

Eleotris tecta Bussing, 1996; Eleotridae: Guab(v)ina (N, $\underline{\mathrm{PS}}, \mathrm{ZN}, \mathrm{CA}, \mathrm{VC})$, Pez perro (PN, PS, ZN, CA), Dormilón (PN, PS, ZN, CA).

Eucinostomus currani Zahuranec, 1980; Gerreidae: Mojarra ( $\underline{\mathrm{PN}}, \underline{\mathrm{PS}}, \mathrm{CA})$, Palmito bandera (PN, PS, CA), Mojarra plateada (PN, PS, CA), Petaca banderita (PN, PS, CA), Petaca banderita del Pacífico (Te).

Eucinostomus melanopterus (Bleeker, 1863); Gerreidae: Mojarra (PN, PS, $\underline{\mathrm{CA}}$ ), Palmito bandera (PN, PS, CA), Mojarra plateada (PN, PS, CA), Petaca banderita (PN, PS, CA), Mojarra de ley (CA), Petaca banderita del Atlántico (Te).

Eugerres brevimanus (Günther, 1864); Gerreidae: Pargo blanco (N, $\underline{\mathrm{PS}})$, Pargo de aleta corta (PN, PS), Mojarra (PN, PS, CA), Mojarra plateada (PN, PS, CA).

Eugerres plumieri (Cuvier, 1830); Gerreidae: Mojarra (PN, PS, CA), Mojarra prieta (CA), Mojarra fina (CA), Mojarra rayada (CA).

Fonchiiichthys uracanthus (Kner, 1863); Loricariidae: Pez diablo (PN, PS, ZN, CA), Plecostomo (PN, PS, ZN, CA, VC, Ac), Guitarra (PS), Zapatero (PS), Pleco (PN, PS, ZN, CA, VC, Ac), Alcalde (PS), Cuchara (PS), Pez gato acorazado (Ac).

Gambusia nicaraguensis Günther, 1866; Poeciliidae: Olomina (PN, PS, ZN, CA, VC), Alumina (PN, PS, ZN, CA, VC), Pepesca (PN, ZN), Olomina de Nicaragua o nicaragüense $(\mathrm{Te})$.

Gobiesox nudus (Linnaeus, 1758); Gobiesocidae: Chupapiedras (PN, PS, $\underline{\mathrm{ZN}}, \underline{\mathrm{CA}}, \mathrm{VC})$, Guarasapo (PN, PS, ZN, CA, VC). 
Gobiomorus dormitor Lacepède, 1800; Eleotridae: Guab(v)ina (PN, PS, ZN, CA, VC), Bocón (PN, PS, ZN, CA), Guab(v)ina del Atlántico (Te).

Gobiomorus maculatus (Günther, 1859); Eleotridae: Guab(v)ina ( $\underline{\mathrm{PN}}, \underline{\mathrm{PS}}, \mathrm{ZN}, \mathrm{CA}, \mathrm{VC})$, Bocón (PN, PS, ZN, CA), Guab(v)ina del Pacífico (Te).

Gobiomorus polylepis Ginsburg, 1953; Eleotridae: Guab(v)ina (N, PS, ZN, CA, VC), Bocón (PN, PS, ZN, CA), Guab(v)ina de escama fina (Te).

Gymnotus cylindricus LaMonte, 1935; Gymnotidae: (Pez) Cuchillo (PN, $\underline{\mathrm{ZN}}, \underline{\mathrm{CA}}, \mathrm{Ac}$ ), (Pez) Navaja (PN, ZN, CA), (Pez) Machete (PN, ZN, CA), Anguila (PN, PS, ZN, CA, VC), Anguila falsa (PN, PS, ZN, CA), Madre de barbudo (ZN, CA), (Pez) Cuchillo redondo $(\mathrm{Te})$.

Gymnotus maculosus Albert y Miller, 1995; Gymnotidae: (Pez) Cuchillo (PN, $\underline{\mathrm{ZN}}, \underline{\mathrm{CA}}$, Ac), (Pez) Navaja (PN, ZN, CA), (Pez) Machete (PN, ZN, CA), Anguila (PN, PS, ZN, CA, VC), Anguila falsa (PN, PS, ZN, CA), Madre de barbudo (ZN, CA), (Pez) Cuchillo manchado (Te).

Hemiancistrus aspidolepis (Günther, 1886); Loricariidae: Pez diablo (PN, $\underline{\mathrm{PS}}, \mathrm{ZN}, \underline{\mathrm{CA}}$ ), Plecostomo (PN, PS, ZN, CA, VC, Ac), Pleco (PN, PS, ZN, CA, VC, Ac), Arrisuaca (PS), Chupapiedras (PS), Pez gato acorazado (Ac).

Hemieleotris latifasciata (Meek y Hidebrand, 1912); Eleotridae: Guab(v)ina (N, $\underline{\text { PS, }}$ ZN, CA, VC), Guab(v)inita (PN, PS).

Hoplias microlepis (Günther, 1864); Erythrinidae: Pez perro (PN, $\underline{P S}, \mathrm{ZN}, \mathrm{CA})$, Pez tigre (PS), Dientón (PS).

Hyphessobrycon panamensis Durbin, 1908; Characidae: Sardina (PN, PS, ZN, CA, VC), Sardinita (PN, PS, ZN, CA), Tetra (VC, Ac), Tetra de Panamá o panameña (Te).

Hyphessobrycon savagei Bussing, 1967; Characidae: Sardina (PN, PS, ZN, CA, VC), Sardinita (PN, PS, ZN, CA), Tetra (VC, Ac), Tetra de Savage (Te).

Hyphessobrycon tortuguerae Böhlke, 1958; Characidae: Sardina (PN, PS, $\underline{\mathrm{ZN}}, \mathrm{CA}, \mathrm{VC})$, Sardinita (PN, PS, ZN, CA), Tetra (VC, Ac), Tetra del Tortuguero (Te).

Hypsophrys nematopus (Günther, 1867); Cichlidae: Moga (PN, $\underline{\mathrm{ZN}}, \underline{\mathrm{CA}})$, Mojarra (PN, PS, $\mathrm{ZN}, \mathrm{CA}, \mathrm{VC})$.

Hypsophrys nicaraguensis (Günther, 1864); Cichlidae: Moga amarilla (PN, $\underline{\mathrm{ZN}}, \underline{\mathrm{CA}}$ ), Moga (PN, ZN, CA), Mojarra (PN, PS, ZN, CA, VC), Mojarra mariposa (ZN, Ac), Cíclido mariposa (Ac), Moga de Nicaragua o nicaraguense (Te).

Imparfinis lineatus (Bussing, 1970); Heptapteridae: Barbudito (S), Barbudo (PN, PS, $\mathrm{ZN}, \mathrm{CA}, \mathrm{VC}$ ), Pez gato (PN, PS, ZN, CA, Ac).

Joturus pichardi Poey, 1860; Mugilidae: Bobo (PN, PS, ZN, $\underline{\mathrm{CA}}$, VC), Boca chica (CA), Lisa de montaña (ZN, CA), Lisa de río (ZN, CA), Sabalete montañero (CA).

Lebiasina boruca (Bussing, 1967); Lebiasinidae: Candela (소), Candelilla (PS).

Lutjanus argentiventris (Peters, 1869); Lutjanidae: Pargo amarillo (Nㅗ $\underline{\mathrm{PS}}, \mathrm{ZN}, \mathrm{CA}$, VC), Boca colorada (PN, PS), Pargo colorado (PN, PS). 
Megalops atlanticus Valenciennes, 1847; Megalopidae: Sábalo (PN, PS, ZN, CA, VC), Sábalo real (PN, PS, ZN, CA), Tarpón (ZN, CA, VC), Tarpón del Atlántico (Te).

Microphis lineatus (Kaup, 1856); Syngnathidae: (Pez) Pipa (PN, PS, $\underline{\mathrm{ZN}}, \underline{\mathrm{CA}}, \mathrm{VC})$, (Pez) Palo (PN, PS, ZN, CA), Trompetero (PN, PS, CA, Ac), (Pez) Aguja (PN, PS, ZN, CA, Ac), (Pez) Lápiz (PN, PS, ZN, CA), Palito (CA), (Pez) Pipa de cola corta (Te).

Mugil cephalus Linnaeus, 1758; Mugilidae: Lisa (PN, PS, ZN, CA, VC), Lisa parda (CA), Pardete (CA).

Mugil curema Valenciennes, 1836; Mugilidae: Lisa ( $\underline{\mathrm{PN}}, \underline{\mathrm{PS}}, \underline{\mathrm{ZN}}, \underline{\mathrm{CA}}, \mathrm{VC})$, Lisa blanca (PN, PS, CA), Lisa criolla (PN, PS, CA), Lisa plateada (PN, PS, CA), Lebrancha (PN, PS).

Odontostilbe dialeptura (Fink y Weitzman, 1974); Characidae: Sardina (PN, PS, ZN, CA, VC), Sardinita (PN, PS, ZN, CA, VC), Tetra (VC, Ac).

Oligoplites palometa (Cuvier, 1831); Carangidae: Jurel (PN, PS, $\underline{\mathrm{ZN}}, \underline{\mathrm{CA}}, \mathrm{VC}),(\mathrm{Pez})$ Cuero (ZN, CA), Zapatero palometa (ZN, CA), Palometa (PN, PS, ZN, CA), Caballa (PN, PS, ZN, CA).

Oligoplites saurus (Bloch y Schneider, 1801); Carangidae: Jurel (PN, PS, ZN, CA, VC), Zapatero (ZN, CA), Zapatero sietecueros (ZN, CA), Piña sietecueros (ZN, CA), Palometa (PN, PS, ZN, CA), Caballa (PN, PS, ZN, CA).

*Oncorhynchus mykiss (Walbaum, 1792); Salmonidae: Trucha (PN, PS, ZN, $\underline{\mathrm{CA}}, \mathrm{VC}$ ), Trucha arcoiris (PN, PS, ZN, CA, VC).

Ophisternon aenigmaticum Rosen y Greenwood, 1976; Synbranchidae: Anguila (PN, PS, $\underline{\mathrm{ZN}}, \mathrm{CA}, \mathrm{VC}$ ), Anguila de pantano (PN, PS, ZN, CA), Anguila de suampo (PN, PS, $\mathrm{ZN}, \mathrm{CA})$, Anguila de lodo (PN, PS, ZN, CA, VC), Anevila (ZN), Anguila falsa (PN, PS, ZN, CA), Culebra (PN, ZN), Anguila de pantano oscura (Te).

*Oreochromis niloticus (Linnaeus, 1758); Cichlidae: Tilapia ( $\underline{\mathrm{PN}}, \underline{\mathrm{PS}}, \underline{\mathrm{ZN}}, \underline{\mathrm{CA}}, \underline{\mathrm{VC}})$, Mojarra (PN, PS, ZN, CA), Tilapia del Nilo (Te).

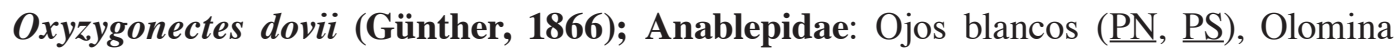
(PN, PS, ZN, CA, VC), Olomina de estero (PN, PS), Alumina (PN, PS, ZN, CA, VC), Killi (Ac).

Parachromis dovii (Günther, 1864); Cichlidae: Guapote (Nㅗ, PS, $\underline{\mathrm{ZN}}, \underline{\mathrm{CA}}, \mathrm{VC})$, Guapote lagunero (PN, ZN, CA), Guapote azul (PN, PS, ZN, CA), Azulón (ZN), Guapotón (PN, ZN), Guapote verde (PN, PS, ZN, CA), Guapote lobo (Ac), Cíclido lobo (Ac).

Parachromis loisellei (Bussing, 1989); Cichlidae: Guapote (PN, PS, ZN, CA, VC), Guapote amarillo (PN, PS, ZN, CA), Guapotillo (PS, ZN, CA), Viejito (ZN), Viejillo (ZN), Guapote dorado (ZN), Guapote de Loisellei (Te).

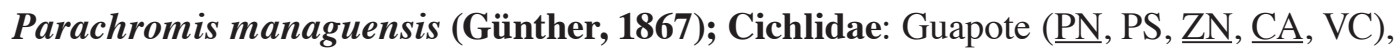
Guapote pinto (PN, PS, ZN, CA), Pinto (ZN, CA), Guapote tigre (PN, PS, ZN, CA, VC, Ac), Barcino $(\mathrm{ZN})$, Guapote jaguar (ZN, CA, Ac), Guapote de Managua o managüense (Te).

Phallichthys amates (Miller, 1907); Poeciliidae: Olomina (PN, PS, $\underline{\mathrm{ZN}}, \underline{\mathrm{CA}}, \mathrm{VC})$, Alumina (PN, PS, ZN, CA, VC), Bubuchita (ZN, CA), Bobita (ZN, CA), Pepesca (PN, ZN). 
Phallichthys quadripunctatus Bussing, 1979; Poeciliidae: Olomina (PN, PS, ZN, CA, VC), Alumina (PN, PS, ZN, CA, VC), Pepesca (PN, ZN).

Phallichthys tico Bussing, 1963; Poeciliidae: Olomina (PN, PS, $\underline{\mathrm{ZN}}, \underline{\mathrm{CA}}, \mathrm{VC}$ ), Alumina (PN, PS, ZN, CA, VC), Pepesca (PN, ZN), Olomina tica (Te).

Pimelodella chagresi (Steindachner, 1876); Heptapteridae: Barbudo (PN, $\underline{\mathrm{PS}}, \mathrm{ZN}, \mathrm{CA}$, VC), Barbudito (PS), Arrechito (PS), Pez gato (VC, Ac), Picalón (PS).

Poecilia gillii (Kner y Steindachner, 1863); Poeciliidae: Panzona ( $\underline{\mathrm{PN}}, \underline{\mathrm{PS}}, \underline{\mathrm{ZN}}, \underline{\mathrm{CA}}, \mathrm{VC})$, Olomina panzona (PN, PS, ZN, CA, VC), Bobita (ZN, CA), Bubucha (ZN, CA), Pipón (CA), Olomina (PN, PS, ZN, CA, VC), Alumina (PN, PS, ZN, CA, VC), Chimbola (PN, ZN), Chompipe (PN, PS), Pepesca (PN, ZN), Molly (Ac), Pepesca panzona (PN, ZN).

Poecilia mexicana Steindachner, 1863; Poeciliidae: Panzona (PN, PS, ZN, $\underline{\mathrm{CA}}, \mathrm{VC})$, Olomina panzona (PN, PS, ZN, CA, VC), Bobita (ZN, CA), Bubucha (ZN, CA), Pipón (CA), Olomina (PN, PS, ZN, CA, VC), Alumina (PN, PS, ZN, CA, VC), Chimbola (PN, $\mathrm{ZN})$, Chompipe (PN, PS), Pepesca (PN, ZN), Pepesca panzona (PN, ZN), Olomina de México o Mexicana (Te).

*Poecilia reticulata Peters, 1859; Poeciliidae: Gupi (PN, PS, ZN, CA, VC, Ac), Olomina (PN, PS, ZN, CA, VC), Alumina (PN, PS, ZN, CA, VC), Gupi reticulado (Te).

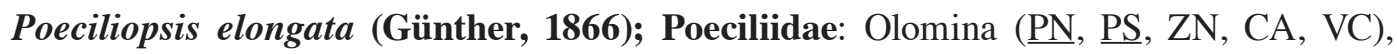
Alumina (PN, PS, ZN, CA, VC), Pepesca (PN, ZN), Olomina elongada (Te).

Poeciliopsis paucimaculata Bussing, 1967; Poeciliidae: Olomina (PN, $\underline{\mathrm{PS}}, \mathrm{ZN}, \mathrm{CA}, \mathrm{VC})$, Olomina manchada (PN, PS, ZN, CA, VC), Alumina (PN, PS, ZN, CA, VC), Pepesca (PN, ZN).

Poeciliopsis retropinna (Regan, 1908); Poeciliidae: Olomina (PN, PS, ZN, CA, VC), Alumina (PN, PS, ZN, CA, VC), Pepesca (PN, ZN).

Poeciliopsis santaelena Bussing, 2008; Poeciliidae: Olomina (N, PS, ZN, CA, VC), Alumina (PN, PS, ZN, CA, VC), Pepesca (PN, ZN), Olomina de Santa Elena (Te).

Poeciliopsis turrubarensis (Meek, 1912); Poeciliidae: Olomina (Nㅗ $\underline{\mathrm{PS}}, \mathrm{ZN}, \mathrm{CA}, \mathrm{VC})$, Alumina (PN, PS, ZN, CA, VC), Pepesca (PN, ZN), Olomina de Turrubares (Te).

Pomadasys bayanus Jordan y Evermann, 1898; Haemulidae: Roncador (PN, PS, ZN, CA, VC), Ronco (PN, PS, ZN, CA), Corocoro (PN, PS, ZN, CA), Corocoro boquimorada (PN, PS), Roncador de río del Pacífico (Te).

Pomadasys crocro (Cuvier, 1830); Haemulidae: Roncador (PN, PS, $\underline{\mathrm{ZN}}, \underline{\mathrm{CA}}, \mathrm{VC}$ ), Ronco (PN, PS, ZN, CA), Burro (ZN, CA), Corocoro (PN, PS, ZN, CA), Corocoro crocró (ZN, CA), Crocró (PN, PS, ZN, CA), Roncador de río del Atlántico (Te).

Priapichthys annectens (Regan, 1907); Poeciliidae: Olomina (Nㅡ, PS, $\underline{\mathrm{ZN}}, \underline{\mathrm{CA}}, \underline{\mathrm{VC}})$, Alumina (PN, PS, ZN, CA, VC), Bubuchita (ZN, CA), Bobita (ZN, CA), Pepesca (PN, $\mathrm{ZN})$.

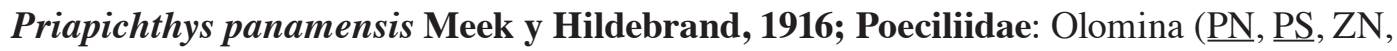
CA, VC), Alumina (PN, PS, ZN, CA, VC), Bubuchita (ZN, CA), Viuda alegre (PS), Pepesca (PN, ZN), Olomina de Panamá o panameña (Te).

Pristis pristis (Linnaeus, 1758); Pristidae: (Pez) Sierra (N, PS, $\underline{\mathrm{ZN}}, \mathrm{CA}, \mathrm{VC})$. 
Pseudocheirodon terrabae Bussing, 1967; Characidae: Sardina (PN, PS, ZN, CA, VC), Sardinita (PN, PS, ZN, CA, VC), Tetra (VC, Ac), Sardinita del Térraba (Te).

Pseudophallus elcapitanensis (Meek y Hidelbrand, 1914); Syngnathidae: (Pez) Pipa ( $\underline{\mathrm{PN}}, \underline{\mathrm{PS}}, \mathrm{ZN}, \mathrm{CA}, \mathrm{VC}),(\mathrm{Pez})$ Palo (PN, PS, ZN, CA), Trompetero (PN, ZN, CA, Ac), (Pez) Aguja (PN, PS, ZN, CA, Ac), (Pez) Lápiz (PN, PS, ZN, CA), Palito (PN, ZN, CA).

Pseudophallus mindi (Meek y Hidelbrand, 1923); Syngnathidae: (Pez) Pipa (PN, PS, $\underline{\mathrm{ZN}}, \underline{\mathrm{CA}}, \mathrm{VC}),(\mathrm{Pez})$ Palo (PN, PS, ZN, CA), Trompetero (PN, ZN, CA, Ac), (Pez) Aguja (PN, PS, ZN, CA, Ac), (Pez) Lápiz (PN, PS, ZN, CA), Palito (PN, ZN, CA).

Pseudophallus starksi (Jordan y Culver, 1895); Syngnathidae: (Pez) Pipa (PN, PS, ZN, $\underline{\mathrm{CA}}, \mathrm{VC}),(\mathrm{Pez})$ Palo (PN, PS, ZN, CA), Trompetero (PN, ZN, CA, Ac), (Pez) Aguja (PN, PS, ZN, CA, Ac), (Pez) Lápiz (PN, PS, ZN, CA), Palito (PN, ZN, CA), (Pez) Pipa de Stark (Te).

Pterobrycon myrnae Bussing, 1974; Characidae: Sardina (PN, PS, ZN, CA, VC), Sardinita (PN, PS, ZN, CA, VC), Tetra (VC, Ac), Tetra semaforo (Ac), Sardinita de Myrna (Te).

Rhamdia guatemalensis (Günther, 1864); Heptapteridae: Barbudo (N, $\underline{\mathrm{PS}}, \underline{\mathrm{ZN}}, \underline{\mathrm{CA}}$, VC), Pinga de/e padre (ZN, CA), Picha de padre (ZN, CA), Chuchín (PN, ZN), Chulín (PN, ZN), Bagre (PN, PS, ZN, CA, VC, Ac), Filín (PN, ZN), Julín (PN, ZN), Pez gato (Ac), Barbudo de barbas largas (Te), Barbudo de Guatemala o guatemalteco (Te).

Rhamdia laticauda (Kner, 1858); Heptapteridae: Barbudo ( $\underline{\mathrm{PN}}, \underline{\mathrm{PS}}, \underline{\mathrm{ZN}}, \underline{\mathrm{CA}}, \underline{\mathrm{VC}})$, Barbudito (PN, PS, ZN, CA, VC), Pinga de/e padre (ZN, CA), Picha de padre (ZN, CA), Chulín (PN, ZN), Bagre (PN, PS, ZN, CA, VC, Ac), Pez gato (Ac), Barbudo de barbas cortas $(\mathrm{Te})$.

Rhamdia nicaraguensis (Günther, 1864); Heptapteridae: Barbudo (Nㅗ, PS, ZN, $\underline{\mathrm{CA}}$, VC), Pinga de/e padre (ZN, CA), Picha de padre (ZN, CA), Chuchín (PN, ZN), Chulín (PN, ZN), Bagre (PN, PS, ZN, CA, VC, AC), Pez gato (Ac), Barbudo de Nicaragua o nicaraguense $(\mathrm{Te})$.

Roeboides bouchellei Fowler, 1923; Characidae: Sardina (PN, PS, ZN, $\underline{\mathrm{ZA}}$, VC), Sardinilla (PN, PS, ZN, CA), Alma seca (PN, PS, ZN, CA), Changuito (PN, PS, ZN, CA), Sardina de vidrio (Te).

Roeboides bussingi Matamoros, Chakrabarty, Angulo, Garita-Alvarado y McMahan, 2013; Characidae: Sardina (PN, PS, ZN, CA, VC), Sardinilla (PN, PS, ZN, CA), Alma seca (PN, PS, ZN, CA), Changuito (PN, PS, ZN, CA), Sardina de vidrio (Te).

Roeboides ilseae Bussing, 1986; Characidae: Sardina (PN, $\underline{P S}, \mathrm{ZN}, \mathrm{CA}, \mathrm{VC})$, Sardinilla (PN, PS, ZN, CA), Alma seca (PN, PS, ZN, CA), Changuito (PN, PS, ZN, CA), Sardina de vidrio $(\mathrm{Te})$.

Sciades guatemalensis (Günther, 1864); Ariidae: Bagre (PN, PS, ZN, CA, VC), Cuminate (PN, PS), Bagre cuatete (PN, PS), Bagre tete (PN, PS), Barbudo (PN, PS, ZN, CA, VC), Pez gato (PN, PS, ZN, CA, VC, Ac), Bagre de Guatemala o guatemalteco (Te).

Sciades seemanni (Günther, 1864); Ariidae: Bagre (N, $\underline{\mathrm{PS}}, \mathrm{ZN}, \mathrm{CA}, \mathrm{VC})$, Cuminate (PN, PS), Cuminate negro (PN, PS), Bagre tete (PN, PS), Barbudo (PN, PS, ZN, CA, $\mathrm{VC}$ ), Pez gato (PN, PS, ZN, CA, VC, Ac). 
Sicydium adelum Bussing, 1996; Gobiidae: Chupapiedras (PN, PS, ZN, CA, VC), Tití (CA), Gobio (PN, PS, ZN, $\underline{\mathrm{CA}}, \mathrm{VC}, \mathrm{Ac}$ ), Gobio de río (PN, PS, ZN, CA, VC, Ac), Lamearenas (PN, PS, ZN, CA).

Sicydium altum Meek, 1907; Gobiidae: Chupapiedras (PN, PS, ZN, CA, VC), Tití (CA), Gobio (PN, PS, $\underline{\mathrm{ZN}}, \underline{\mathrm{CA}}, \mathrm{VC}, \mathrm{Ac}$ ), Gobio de río (PN, PS, ZN, CA, VC, Ac), Lamearenas (PN, PS, ZN, CA).

Sicydium salvini Ogilvie-Grant, 1884; Gobiidae: Chupapiedras (PN, PS, ZN, CA, VC), Tití (CA), Gobio (Nㅗ PS, ZN, CA, VC, Ac), Gobio de río (PN, PS, ZN, CA, VC, Ac), Lamearenas (PN, PS, ZN, CA).

Synbranchus marmoratus Bloch, 1795; Synbranchidae: Anguila (N, $\underline{\mathrm{PS}}, \underline{\mathrm{ZN}}, \underline{\mathrm{CA}}$, $\underline{\mathrm{VC}}$ ), Anguila de pantano (PN, PS, ZN, CA), Anguila de suampo (PN, PS, ZN, CA), Anguila de lodo (PN, PS, ZN, CA, VC), Anevila (ZN), Anguila falsa (PN, PS, ZN, CA), Culebra (PN, ZN), Anguila de pantano moteada (Te).

Tomocichla sieboldii (Kner, 1863); Cichlidae: Vieja (PN, PS, ZN, CA, VC), Moga verde (PN, PS, ZN, CA, Ac), Mojarra (PN, PS, ZN, CA, VC).

Tomocichla tuba (Meek, 1912); Cichlidae: Vieja (PN, PS, ZN, $\underline{\mathrm{CA}}$, VC), Moga verde (PN, PS, ZN, CA, Ac), Tuba (ZN, CA, Ac), Mojarra (PN, PS, ZN, CA, VC).

Trichomycterus striatus (Meek y Hidelbrand, 1913); Trichomycteridae: Barbudo (PN, $\underline{\mathrm{PS}}, \mathrm{ZN}, \mathrm{CA}, \mathrm{VC})$, Barbudito (PN, PS, ZN, CA, VC), Laucha (PS), Babosa (PS), Pez gato lápiz (Ac).

Trinectes fonsecensis (Günther, 1862); Achiridae: Lenguado (N, $\underline{\mathrm{PS}}, \mathrm{ZN}, \mathrm{CA}, \mathrm{VC})$, Suela (PN, PS, CA), Lenguado redondo (PN, PS, CA), Suela rayada (PN, PS), (Pez) Hoja (PN, PS, CA), Suela de cola manchada (Te).

Trinectes paulistanus (Miranda Ribeiro, 1915); Achiridae: Lenguado (PN, PS, $\underline{\mathrm{ZN}}, \underline{\mathrm{CA}}$, VC), Suela (PN, PS, CA), Lenguado redondo (PN, PS, CA), (Pez) Hoja (PN, PS, CA).

Vieja maculicauda (Regan, 1905); Achiridae: Vieja ( $\underline{\mathrm{ZN}}, \underline{\mathrm{CA}})$, Mojarra (PN, PS, ZN, CA, VC), Pis pis (ZN, CA), Mojarra de estero (ZN, CA), Palometa (ZN, CA), Mojarra azul (ZN, CA).

Xenophallus umbratilis (Meek, 1912); Poeciliidae: Olomina (N, PS, ZN, $\underline{\mathrm{CA}}, \mathrm{VC})$, Alumina (PN, PS, ZN, CA, VC), Pepesca (PN, ZN).

\section{Lista de nombres comunes y técnicos de los peces de agua dulce de Costa Rica}

Se presenta a continuación un listado, alfabéticamente organizado, que incluye 260 nombres comunes y 60 nombres técnicos. Se indica en superíndice el origen, ya sea popular, común o vernacular (Co) o técnico o vulgar $(\mathrm{Te})$ y, cuando se conoce, el significado, ya sea en alusión a un rasgo físico o morfológico $(\mathrm{Fi})$, de coloración $(\mathrm{Cl})$, de comportamiento $(\mathrm{Cm})$, de uso de hábitat (Ha) o asociado a algún tipo de sonido producido por la(s) especie(s) en cuestión (Onomatopéyico; On), en alusión a un área geográfica $(\mathrm{Ge})$ o en honor a una personalidad del ámbito científico o político $(\mathrm{Pe})$. Se mencionan, de forma ordenada en correspondencia a la frecuencia de uso, el nombre científico de las distintas especies a las cuales se les asigna el nombre en cuestión. Para cada nombre común, de esta manera, la especie mencionada de primero en la lista es aquella a la cual, con mayor frecuencia relativa, debido en parte a 
diferentes parámetros tales como su abundancia en ambientes naturales, uso e importancia económica, frecuencia de captura (para el caso de especies de interés socio-económico), entre otros, se le asigna comúnmente el nombre en cuestión. Para el caso de nombres comunes aplicados de manera indistinta a diferentes especies dentro de un mismo género o familia los nombres científicos se mencionan en orden alfabético. Cuando dos o más especies pertenecientes a un mismo género se mencionan de forma consecutiva, o en un mismo párrafo, por convención, el nombre genérico se escribe completamente solo la primera vez, en casos subsiguientes se utiliza solo la letra inicial, en mayúscula. La información que se presenta a continuación complementa el listado anterior, por tanto se sugiere cruzar referencias.

Aguja (Pez) ${ }^{\mathrm{Co}, \mathrm{Fi}}$ : miembros de la familia Syngnathidae: Microphis lineatus, Pseudophallus elcapitanensis, $P$. mindii y $P$. starksi.

Alcalde $\mathrm{Co}, \mathrm{Cm}$ : Fonchiiichthys uracanthus, Loricariidae.

Alfiler ${ }^{\mathrm{Co}}$, Fi: miembros de la familia Atherinopsidae: Atherinella argentea, A. chagresi, A. hubbsi, A. milleri y A. sardina.

Alma seca ${ }^{\mathrm{Co}}, \mathrm{Fi} / \mathrm{Cl}$ : Roeboides bouchellei, R. bussingi y R. ilseae, Characidae.

Alumina $\mathrm{Co}_{\mathrm{Fi}} \mathrm{Cl}$ : miembros de las familias Poeciliidae: Alfaro cultratus, Brachyrhaphis cascajalensis, B. holdridgei, B. olomina, B. parismina, B. rhabdophora, B. roseni, $B$. terrabensis, Gambusia nicaraguenses, Phallichthys amates, P. quadripunctatus, P. tico, Poecilia gillii, P. mexicana, P. reticulata, Poeciliopsis elongata, $P$. paucimaculata, P. retropinna, P. santaelena, P.turrubarensis, Priapichthys annectens, P. panamensis y Xenophallus umbratilis, Anablepidae: Oxyzygonectes dovii, y Rivulidae: Cynodonichthys fuscolineatus, C. glaucus, C. hildebrandi, C. isthmensis, C. rubripunctatus, $C$. siegfriedi y $C$. uroflammeus.

-picuda Co, Fi: Belonesox belizanus, Poeciliidae.

Anevila Co, Fi: Synbranchus marmoratus y Ophisternon aenigmaticum, Synbranchidae.

Anguila Co, Fi: Anguilla rostrata, Anguillidae, Synbranchus marmoratus, Ophisternon aenigmaticum, Synbranchidae, Gymnotus maculosus, Gymnotus cylindricus, Gymnotidae, y Brachyhypopomus occidentalis, Hypopomidae.

-Americana Te, Ge: Anguilla rostrata, Anguillidae.

-de lodo ${ }^{\mathrm{Co}, \mathrm{Ha}}$ : Synbranchus marmoratus y Ophisternon aenigmaticum, Synbranchidae. -de mar Co, Ha: Anguilla rostrata, Anguillidae.

-pantano Co, Ha: Synbranchus marmoratus y Ophisternon aenigmaticum, Synbranchidae.

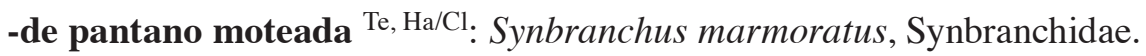

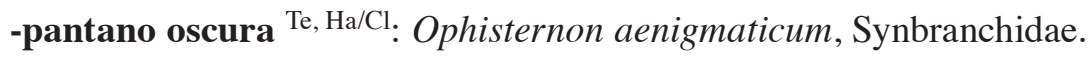

-de suampo Co, Ha: Synbranchus marmoratus y Ophisternon aenigmaticum, Synbranchidae.

-falsa Co, Fi: Synbranchus marmoratus, Ophisternon aenigmaticum, Synbranchidae, Gymnotus maculosus, Gymnotus cylindricus, Gymnotidae, y Brachyhypopomus occidentalis, Hypopomidae.

-verdadera ${ }^{\mathrm{Co}}$, Fi: Anguilla rostrata, Anguillidae. 
Arrechito $\mathrm{Co}, \mathrm{Cm}$ : Pimelodella chagresi, Heptapteridae.

Arrisuaca $\mathrm{Co}, \mathrm{Cm}$ : Hemiancistrus aspidolepis, Loricariidae.

Azulón ${ }^{\mathrm{Co}, \mathrm{Cl}}$ : Parachromis dovii, Cichlidae.

Babosa ${ }^{\mathrm{Co}, \mathrm{Fi}}$ : Trichomycterus striatus, Trichomycteridae.

Bagre Co: Sciaedes guatemalensis, S. seemanni, Cathorops steindachneri y C. tuyra, Ariidae, Rhamdia guatemalensis, $R$. nicaraguensis y $R$. laticauda, Heptapteridae.

-baboso ${ }^{\mathrm{Co}}, \mathrm{Fi}$ : Cathorops steindachneri, Ariidae.

-besucón Co, Fi: Cathorops tuyra, Ariidae.

-cuatete ${ }^{\mathrm{Co}}$ : Sciaedes guatemalensis, Ariidae.

-de Guatemala, guatemalteco ${ }^{\mathrm{Te}}$, Ge: Sciaedes guatemalensis, Ariidae.

-de Steindachner Te, Pe: Cathorops steindachneri, Ariidae.

-tete Co: Sciaedes guatemalensis y S. seemanni, Ariidae.

Banana $\mathrm{Co}, \mathrm{Fi} / \mathrm{Cl}$ : Awaous banana, Gobiidae.

Barbudito Co, Fi: Imparfinis lineatus, Pimelodella chagresi, Rhamdia laticauda, Heptapteridae, y Trichomycterus striatus, Trichomycteridae.

Barbudo ${ }^{\mathrm{Co}}$, $\mathrm{Fi}$ : Rhamdia guatemalensis, R. nicaraguensis, R. laticauda, Imparfinis lineatus, Pimelodella chagresi, Heptapteridae, Sciaedes guatemalensis, S. seemanni, Cathorops steindachneri, C. tuyra, Ariidae, y Trichomycterus striatus, Trichomycteridae.

-de barbas cortas ${ }^{\mathrm{Te}}$, Fi. Rhamdia laticauda, Heptapteridae.

-de barbas largas ${ }^{\mathrm{Te}}$, Fi: Rhamdia guatemalensis, Heptapteridae.

-de Guatemala, guatemalteco ${ }^{\mathrm{Te}}, \mathrm{Ge}$ : Rhamdia guatemalensis, Heptapteridae.

-de Nicaragua, nicaragüense ${ }^{\mathrm{Te}}$, Ge: Rhamdia nicaraguensis, Heptapteridae.

Barcino ${ }^{\mathrm{Co}} \mathrm{Cl}^{\mathrm{C}}$ : Parachromis managuensis, Cichlidae.

Bobita Co, Fi: Poecilia gillii, P. mexicana, Phallichthys amates y Priapichthys annectens, Poeciliidae.

Bobo ${ }^{\mathrm{Co}}$, Fi: Joturus pichardi, Mugilidae.

Boca chica ${ }^{\mathrm{Co}}$, Fi. Joturus pichardi, Mugilidae.

Boca colorada ${ }^{\mathrm{Co}}, \mathrm{Cl}$ : Lutjanus argentiventris, Lutjanidae.

Bocón Co, Fi: Gobiomorus dormitor, G. maculatus y G. polylepis, Eleotridae.

Bonito ${ }^{\mathrm{Co}}, \mathrm{Cl}$ : Caranx caballus, C. caninus y C. latus, Carangidae.

Bubucha Co, Fi: Poecilia gillii y P. mexicana, Poeciliidae.

-rayada $\mathrm{Co}, \mathrm{Cl}$ : Poeciliopsis turrubarensis, Poecilidae.

Bubuchita Co, Fi: Poecilia gillii, P. mexicana, Phallichthys amates y Priapichthys annectens, Poeciliidae.

Burra Co, Cm: Amatitlania siquia y A. kanna, Cichlidae.

Caballa Co: Caranx caballus, C. caninus, C. latus, Oligoplites palometa y O. saurus, Carangidae. 
Calva ${ }^{\mathrm{Co}}, \mathrm{Cl}$ : Centropomus parallelus, Centropomidae.

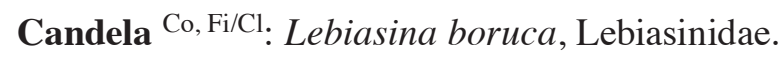

Candelilla ${ }^{\mathrm{Co}, \mathrm{Fi} / \mathrm{Cl}}$ : Lebiasina boruca, Lebiasinidae.

Capani ${ }^{\mathrm{Co}}$ : Cyphocharax magdalenae, Curimatidae.

Capaniz ${ }^{\mathrm{Co}}$ : Cyphocharax magdalenae, Curimatidae.

Carate ${ }^{\mathrm{Co}}, \mathrm{Cl}_{1}$ : Amphilophus longimanus, A. rostratus, Amatitlania siquia y A. kanna, Cichlidae.

Carpa Co: Cyphocharax magdalenae, Curimatidae. Este nombre se utiliza también para nombrar varias especies de peces exóticas miembros de la familia Cyprinidae.

Changuito ${ }^{\mathrm{Co}}$ : Roeboides bouchellei, R. ilseae y R. bussingi, Characidae.

Chimbembe ${ }^{\mathrm{Co}}$ : Cathorops steindachneri y C. tuyra, Ariidae.

Chimbola ${ }^{\mathrm{C}}$, Fi: Poecilia gillii y P. mexicana, Poeciliidae.

Chogorra Co: Andinoacara coeruleopunctatus, Cryptoheros myrnae, Cryptoheros sajica, Amatitlania siquia y A. kanna, Cichlidae.

Cholesca ${ }^{\mathrm{Co}}$ : Amphilophus longimanus y Archocentrus multispinosus, Cichlidae.

Chompipe Co, Fi. Poecilia gillii y P. mexicana, Poeciliidae.

Chuchín Co, Fi: Rhamdia guatemalensis y R. nicaraguensis, Heptapteridae.

Chulín Co: Rhamdia guatemalensis, R. nicaraguensis y R. laticauda, Heptapteridae.

Chumbo ${ }^{\mathrm{Co}}$ : Caranx caballus, Carangidae.

Chupapiedras Co, Cm: Sicydium salvini, S. altum y S. adelum, Gobiidae.

Cocinero ${ }^{\mathrm{Co}}$ : Caranx caballus, Carangidae.

Cojinoa ${ }^{\mathrm{Co}}$ : Caranx caballus, Carangidae.

Cojinúa chata ${ }^{\mathrm{Co}}$, Fi: Caranx caballus, Carangidae.

Cojinúa ${ }^{\mathrm{Co}}$ : Caranx caballus, Carangidae.

Coliroja $\mathrm{Co}, \mathrm{Cl}$ : Astyanax aeneus, Characidae.

Congo $\mathrm{Co}, \mathrm{Cl} / \mathrm{Cm}$ : Amatitlania siquia y A. kanna, Cichlidae.

Convicto ${ }^{\mathrm{Co}, \mathrm{Cl}}$ : Amatitlania siquia y A. kanna, Cichlidae.

Corocoro Co, On: Pomadasys bayanus y P. crocro, Haemulidae.

-boquimorada Co, Fi: Pomadasys bayanus, Haemulidae.

-crocró Co, On: Pomadasys crocro, Haemulidae.

Crocró Co, On: Pomadasys crocro, Haemulidae.

Cuchara ${ }^{\mathrm{Co}, \mathrm{Fi}}$ : Fonchiiichthys uracanthus, Loricariidae.

Cuchillo (Pez) ${ }^{\mathrm{Co}, \mathrm{Fi}}$ : Gymnotus maculosus, G. cylindricus, Gymnotidae, y Brachyhypopomus occidentalis, Hypopomidae.

-aplanado Te, Fi: Brachyhypopomus occidentalis, Hypopomidae.

-manchado ${ }^{\mathrm{Te}}, \mathrm{Cl}$ : Gymnotus maculosus, Gymnotidae.

-redondo Te, Fi: Gymnotus cylindricus, Gymnotidae. 
Cuero (Pez) Co, Fi: Oligoplites palometa, Carangidae.

Culebra ${ }^{\mathrm{Co}}$, Fi: Synbranchus marmoratus y Ophisternon aenigmaticum, Synbranchidae.

Cuminate Co: Sciaedes guatemalensis, S. seemanni, Cathorops steindachneri y C. tuyra, Ariidae.

-baboso Co, Fi: Cathorops steindachneri, Ariidae.

-besucón Co, Fi: Cathorops tuyra, Ariidae.

-negro ${ }^{\mathrm{Co}} \mathrm{Cl}$ : Sciaedes seemanni, Ariidae.

Dientón Co, Fi: Hoplias microlepis, Erythrinidae.

Dormilón Co, Cm: Dormitator latifrons, D. maculatus, Eleotris amblyopsis, E. pisonis, E. tecta y E. picta, Eleotridae.

-manchado ${ }^{\mathrm{Co}, \mathrm{Cl}}$ : Eleotris picta, Eleotrodae.

Filín Co, Fi: Rhamdia guatemalensis, Heptapteridae.

Gaspar Co: Atractosteus tropicus, Lepisosteidae.

-tropical Co, Ge: Atractosteus tropicus, Lepisosteidae.

Gasparcillo ${ }^{\mathrm{Co}, \mathrm{Fi}}$ : Belonesox belizanus, Poeciliidae.

Gobio Co: miembros de la familia Gobiidae: Awaous banana, A. transandeanus, Sicydium adelum, S. altum y S. salvini.

-de río Co, Ha. miembros de la familia Gobiidae: Awaous banana, A. transandeanus, Sicydium adelum, S. altum y S. salvini.

Golosa $\mathrm{Co}, \mathrm{Cm}$ : Astyanax aeneus, Characidae.

Guab(v)ina Co: miembros de las familias Eleotridae: Dormitator latifrons, D. maculatus, Eleotris amblyopsis, E. pisonis, E. tecta, E. picta, Gobiomorus dormitor, G. maculatus, G. polylepis y Hemieleotris latifasciata, y Gobiidae: Awaous banana y A. transandeanus.

Guab(v)inita ${ }^{\mathrm{Co}}$ : Hemieleotris latifasciata, Eleotridae.

Guajale Co: Centropomus medius, Centropomidae.

-aleta manchada $\mathrm{Co}, \mathrm{Cl}$ : Centropomus medius, Centropomidae.

-del Atlántico ${ }^{\mathrm{Te}, \mathrm{Ge}}$ : Centropomus pectinatus, Centropomidae.

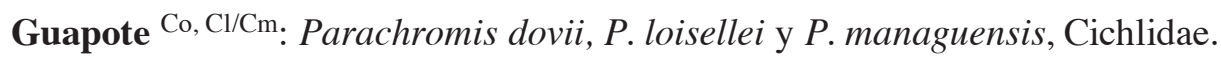

-amarillo $\mathrm{Co}^{\mathrm{Cl}}$ : Parachromis loisellei, Cichlidae.

-azul ${ }^{\mathrm{Co}}, \mathrm{Cl}$ : Parachromis dovii, Cichlidae.

-de Loiselle Te, Pe: Parachromis loisellei, Cichlidae.

-de Managua, managüense ${ }^{\mathrm{Te}, \mathrm{Ge}}$ : Parachromis managuensis, Cichlidae.

-dorado ${ }^{\mathrm{Co}, \mathrm{Cl}}$ : Parachromis loisellei, Cichlidae.

-jaguar $\mathrm{Co}, \mathrm{Cl} / \mathrm{Cm}$ : Parachromis managuensis, Cichlidae.

-lagunero Co, Ha. Parachromis dovii, Cichlidae.

-pinto ${ }^{\mathrm{Co}, \mathrm{Cl}}$ : Parachromis managuensis, Cichlidae. 
-tigre ${ }^{\mathrm{Co}, \mathrm{Cl}}$ : Parachromis managuensis, Cichlidae.

-verde ${ }^{\mathrm{Co}, \mathrm{Cl}}$ : Parachromis dovii, Cichlidae.

Guapotón ${ }^{\mathrm{Co}}$, Fi: Parachromis dovii, Cichlidae.

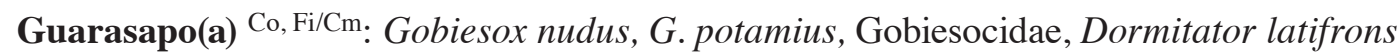
y D. maculatus, Eleotridae.

Guitarra Co, Fi. Fonchiiichthys uracanthus, Loricariidae.

Gupi Co: Poecilia reticulata, Poeciliidae.

-reticulado ${ }^{\mathrm{Te}}, \mathrm{Cl}$ : Poecilia reticulata, Poeciliidae.

Hoja (Pez) Co, Fi: miembros de las familias Achiridae: Achirus mazatlanus, Trinectes fonsecensis y T. paulistanus, y Paralichthyidae: Citharicthys spilopterus y C. uhleri.

Jack Co: Caranx latus, Carangidae.

Julín Co, Fi. Rhamdia guatemalensis, Heptapteridae.

Jurel Co: miembros de la familia Carangidae: Caranx caballus, C. caninus, C. latus, Oligoplites palometa y O. saurus.

-blanco ${ }^{\mathrm{Co}, \mathrm{Cl}}$ : Caranx latus, Carangidae.

-coliamarilla/de cola amarilla ${ }^{\mathrm{Co}} \mathrm{Cl}$ : Caranx latus, Carangidae.

-del Pacífico Te, Ge: Caranx caninus, Carangidae.

-ojón Co, Fi: Caranx caninus y C. latus, Carangidae.

-toro $\mathrm{Co}, \mathrm{Cl} / \mathrm{Cm}$ : Caranx caninus, Carangidae.

-verde ${ }^{\mathrm{Co}}, \mathrm{Cl}$ : Caranx caballus, Carangidae.

Killi Co: miembros de las familias Rivulidae: Cynodonichthys fuscolineatus, C. glaucus, C. hildebrandi, C. isthmensis, C. rubripunctatus, C. siegfriedi, C. uroflammeus, y Anablepidae: Oxyzygonectes dovii.

Lamearenas Co, Cm: Awaous banana, A. transandeanus, Sicydium salvini, S. altum y S. adelum, Gobiidae.

Lápiz (Pez) ${ }^{\mathrm{Co}}$ Fi: miembros de la familia Syngnathidae: Microphis lineatus, Pseudophallus elcapitanensis, $P$. mindii y $P$. starksi.

Laucha ${ }^{\mathrm{Co}}$, Fi: Trichomycterus striatus, Trichomycteridae.

Lebrancha ${ }^{\text {Co: }}$ Mugil curema, Mugilidae.

Lenguado Co, Fi: miembros de las familias Achiridae: Achirus mazatlanus, Trinectes fonsecensis y T. paulistanus, y Paralichthyidae: Citharicthys spilopterus y C. uhleri.

-redondo Co, Fi: miembros de la familia Achiridae: Achirus mazatlanus, Trinectes fonsecensis y T. paulistanus.

Lisa $\mathrm{Co}, \mathrm{Fi} / \mathrm{Cl}$ : Mugil curema, M. cephalus y Agonostomus monticola, Mugilidae.

-blanca ${ }^{\mathrm{Co}}, \mathrm{Cl}$ : Mugil curema, Mugilidae.

-criolla ${ }^{\mathrm{Co}, \mathrm{Ge}}$ : Mugil curema, Mugilidae.

-de montaña Co, Ha: Agonostomus monticola y Joturus pichardi, Mugilidae. -de río Co, Ha: Agonostomus monticola y Joturus pichardi, Mugilidae. 
-parda ${ }^{\mathrm{Co}}, \mathrm{Cl}$ : Mugil cephalus, Mugilidae.

-plateada ${ }^{\mathrm{Co}}, \mathrm{Cl}$ : Mugil curema, Mugilidae.

Lobo (Cíclido) ${ }^{\mathrm{Co}}, \mathrm{Cm}$ : Parachromis dovii, Cichlidae.

Lucia ${ }^{\mathrm{Co}}$ : Eleotris amblyopsis, Eleotridae.

Machaca Co: Brycon costaricensis, B. behreae y Astyanax aeneus, Characidae.

-del Atlántico ${ }^{\mathrm{Te}}, \mathrm{Ge}$ : Brycon costaricensis, Characidae.

-del Pacífico Te, Ge: Brycon behreae, Characidae.

Machete (Pez) ${ }^{\mathrm{Co}, \mathrm{Fi}}$ : Gymnotus maculosus, G. cylindricus, Gymnotidae, y Brachyhypopomus occidentalis, Hypopomidae.

Machín ${ }^{\mathrm{Co}}, \mathrm{Cl}$ : Agonostomus monticola, Mugilidae.

Madre de barbudo Co, Fi/Cm: Gymnotus maculosus, G. cylindricus, Gymnotidae, y Brachyhypopomus occidentalis, Hypopomidae.

Madrecazón Co: Carcharhinus leucas, Carcharhinidae.

Masamiche Co: Amphilophus rostratus, Cichlidae.

Mechín Co: Agonostomus monticola, Mugilidae.

Midas (Cíclido) ${ }^{\mathrm{Co}} \mathrm{Cm}$ : Amphilophus citrinellus, Cichlidae.

Moga Co: Hypsophrys nematopus y H. nicaraguensis, Cichlidae.

-amarilla ${ }^{\mathrm{Co}}, \mathrm{Cl}$ : Hypsophrys nicaraguensis, Cichlidae.

-de Nicaragua, nicaraguense Te, Ge: Hypsophrys nicaraguensis, Cichlidae.

-verde ${ }^{\mathrm{Co}}, \mathrm{Cl}$ : Tomocichla tuba y T. sieboldii, Cichlidae.

Mojarra ${ }^{\mathrm{Co}}$ : miembros de las familias Cichlidae: Amatitlania kanna, A. siquia, Amphilophus alfari, A altifrons, A. bussingi, A. citrinellus, A. diquis, A. longimanus, A. lyonsi, A. rhytisma, A. rostratus, Andinoacara coeruleopunctatus, Archocentrus centrarchus, A. multispinosus, Cryptoheros myrnae, C. sajica, C. septemfasciatus, Hypsophrys nematopus, H. nicaraguensis, Oreochromis niloticus, Tomocichla sieboldii, T. tuba y Vieja maculicauda, y Gerreidae: Diapterus rhombeus, Eucinostomus currani, E. melanopterus, Eugerres brevimanus y E. plumieri.

-amarilla $\mathrm{Co}, \mathrm{Cl}$ : Amphilophus rostratus, Cichlidae.

-arcoiris ${ }^{\mathrm{Co}}, \mathrm{Cl}$ : Archocentrus multispinosus Cichlidae.

-azul Co, $\mathrm{Cl}$ : Vieja maculicauda, Cichlidae.

-caitipia Co: Diapterus rhombeus, Gerreidae.

-de Alfaro Te, Pe: Amphilophus alfari, Cichlidae.

-de Bussing Te, Pe: Amphilophus bussingi, Cichlidae.

-de estero Co, Ha: Vieja maculicauda, Cichlidae, y Diapterus rhombeus, Gerreidae.

-de ley Co: Eucinostomus melanopterus, Gerreidae.

-de Myrna Te, Pe. Cryptoheros myrnae, Cichlidae.

-de pecho rojo ${ }^{\mathrm{Co}, \mathrm{Cl}}$ : Amphilophus longimanus, Cichlidae.

-del Diquis Te, Ge: Amphilophus diquis, Cichlidae. 
-dorada ${ }^{\mathrm{Co}}, \mathrm{Cl}$ : Amphilophus rostratus, Cichlidae.

-fina ${ }^{\mathrm{Co}}$, Fi: Eugerres plumieri, Gerreidae.

-mariposa ${ }^{\mathrm{Co}}, \mathrm{Cl}$ : Hypsophrys nicaraguensis, Cichlidae.

-Midas Co, Cm: Amphilophus citrinellus, Cichlidae.

-pastel ${ }^{\mathrm{Co}, \mathrm{Cl}}$ : Amphilophus alfari, Cichlidae.

-perla ${ }^{\mathrm{Co}}, \mathrm{Cl}$ : Amphilophus rhytisma, Cichlidae.

-picuda Co, Fi. Amphilophus rostratus, Cichlidae.

-plateada Co, Cl: Diapterus rhombeus, Eucinostomus currani, E. melanopterus y Eugerres brevimanus, Gerreidae.

-prieta ${ }^{\mathrm{Co}, \mathrm{Cl}}$ : Eugerres plumieri, Gerreidae.

-rayada ${ }^{\mathrm{Co}}, \mathrm{Cl}$ : Amatitlania kanna, A, siquia, Archocentrus centrarchus, Cichlidae, y Eugerres plumieri, Gerreidae.

-real ${ }^{\mathrm{Co}}, \mathrm{Cl}$ : Amphilophus bussingi y Amphilophus rhytisma, Cichlidae.

-topaz ${ }^{\mathrm{Co}, \mathrm{Cl}}$ : Cryptoheros myrnae, Cichlidae.

Mojarrita Co, Fi: Amatitlania kanna, A. siquia y Andinoacara coeruleopunctatus, Cichlidae.

Navaja (Pez) Co, Fi: Gymnotus maculosus, G. cylindricus, Gymnotidae, y Brachyhypopomus occidentalis, Hypopomidae.

Ojos blancos ${ }^{\mathrm{Co}}, \mathrm{Cl}$ : Oxyzygonectes dovii, Anablepidae.

Olomina $\mathrm{Co}, \mathrm{Fi} / \mathrm{Cl}$ : miembros de las familias Poeciliidae: Alfaro cultratus, Brachyrhaphis cascajalensis, B. holdridgei, B. olomina, B. parismina, B. rhabdophora, B. roseni, $B$. terrabensis, Gambusia nicaraguenses, Phallichthys amates, P. quadripunctatus, $P$. tico, Poecilia gillii, $P$. mexicana, $P$. reticulata, Poeciliopsis elongata, $P$. paucimaculata, P. retropinna, P. santaelena, P.turrubarensis, Priapichthys annectens, P. panamensis y Xenophallus umbratilis, Anablepidae: Oxyzygonectes dovii y Rivulidae: Cynodonichthys fuscolineatus, C. glaucus, C. hildebrandi, C. isthmensis, C. rubripunctatus, $C$. siegfriedi y C. uroflammeus.

-azul Te, $\mathrm{Cl}$ : Cynodonichthys glaucus, Rivulidae.

-de Alfaro Te, Pe. Alfaro cultratus, Poeciliidae.

-de estero ${ }^{\mathrm{Co}, \mathrm{Ha}}$ : Oxyzygonectes dovii, Anablepidae.

-de Hildebrand Te, Pe: Cynodonichthys hildebrandi, Rivulidae.

-de Holdridge Te, Pe: Brachyrhaphis holdridgei, Poeciliidae.

-de México o mexicana Te, Ge: Poecilia mexicana, Poeciliidae.

-de Nicaragua o nicaragüense Te, Ge: Gambusia nicaraguenses, Poeciliidae.

-de Panamá o panameña Te, Ge: Priapichthys panamensis, Poeciliidae.

-de Rosen Te, Pe: Brachyrhaphis roseni, Poeciliidae.

-de Santa Elena Te, Ge: Poeciliopsis santaelena, Poeciliidae.

-de Siegfried Co, Pe: Cynodonichthys siegfriedi, Rivulidae.

-de Turrubares Te, Ge: Poeciliopsis turrubarensis, Poeciliidae. 
-del Arenal Te, Ge: Cynodonichthys fuscolineatus, Rivulidae.

-del Cascajal Te, Ge: Brachyrhaphis cascajalensis, Poeciliidae.

-del Parismina Te. Brachyrhaphis parismina, Poeciliidae.

-del Térraba Te, Ge: Brachyrhaphis terrabensis, Poeciliidae.

-elongada $\mathrm{Te}, \mathrm{Fi}$ : Poeciliopsis elongata, Poeciliidae.

-flama ${ }^{\mathrm{Te}}, \mathrm{Cl}$ : Cynodonichthys uroflammeus, Rivulidae.

-manchada ${ }^{\mathrm{Co}, \mathrm{Cl}}$ : Poeciliopsis paucimaculata, Poeciliidae.

-moteada ${ }^{\mathrm{Te}, \mathrm{Cl}}$ : Cynodonichthys hildebrandi, Rivulidae.

-panzona Co, Fi. Poecilia gillii y P. mexicana, Poeciliidae.

-picuda ${ }^{\mathrm{Co}} \mathrm{Pi}$ : Belonesox belizanus, Poeciliidae.

-tica Te, Ge: Phallichthys tico, Poeciliidae.

Palito Co, Fi: miembros de la familia Syngnathidae: Microphis lineatus, Pseudophallus elcapitanensis, P. mindii y P. starksi.

Palmito ${ }^{\mathrm{Co}}, \mathrm{Cl}$ : Diapterus rhombeus, Gerreidae.

-bandera ${ }^{\mathrm{Co}}, \mathrm{Cl}:$ Eucinostomus currani y Eucinostomus melanopterus, Gerreidae.

Palo (Pez) ${ }^{\mathrm{C}}$, Fi. miembros de la familia Syngnathidae: Microphis lineatus, Pseudophallus elcapitanensis, P. mindii y P. starksi.

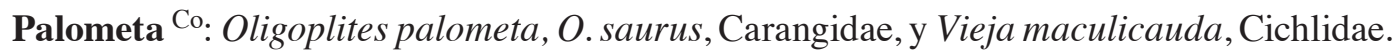

Panzona Co, Fi: Poecilia gillii y P. mexicana, Poeciliidae.

Pardete $^{\mathrm{Co}, \mathrm{Cl}}$ : Mugil cephalus, Mugilidae.

Pargo Co: Lutjanus argentiventris, Lutjanidae, y Eugerres brevimanus, Gerreidae.

-amarillo ${ }^{\mathrm{Co}, \mathrm{Cl}}$ : Lutjanus argentiventris, Lutjanidae.

-blanco ${ }^{\mathrm{Co}, \mathrm{Cl}}$ : Eugerres brevimanus, Gerreidae.

-colorado $\mathrm{Co}^{\mathrm{Cl}}$ : Lutjanus argentiventris, Lutjanidae.

-de aleta corta $\mathrm{Co}, \mathrm{Fi}$ : Eugerres brevimanus, Gerreidae.

Pecho rojo ${ }^{\mathrm{Co}, \mathrm{Cl}}$ : Amphilophus longimanus, Cichlidae.

Pejelagarto ${ }^{\mathrm{Co}}$, $\mathrm{Fi}$ : Atractosteus tropicus, Lepisosteidae.

Pejerrey ${ }^{\mathrm{Co}}, \mathrm{Fi}$ : miembros de la familia Atherinopsidae: Atherinella argentea, A. chagresi, A. hubbsi, A. milleri y A. sardina.

-de Hubbs Te, Pe: Atherinella hubbsi, Atherinopsidae.

-de Miller Te, Pe: Atherinella milleri, Atherinopsidae.

-del Chagres $\mathrm{Te}, \mathrm{Ge}$ : Atherinella chagresi, Atherinopsidae.

Pepesca Co: miembros de la familia Poeciliidae: Alfaro cultratus, Belonesox belizanus, Brachyrhaphis cascajalensis, B. holdridgei, B. parismina, B. rhabdophora, B. roseni, B. terrabensis, Gambusia nicaraguenses, Phallichthys amates, P. quadripunctatus, P. tico, Poecilia gillii, P. mexicana, Poeciliopsis elongata, P. paucimaculata, $P$. retropinna, $P$. santaelena, $P$. turrubarensis, Priapichthys annectens, $P$. panamensis y Xenophallus umbratilis. 
-gaspar Co, Fi: Belonesox belizanus, Poeciliidae.

-panzona Co, Fi. Poecilia gillii y P. mexicana, Poeciliidae.

Petaca banderita ${ }^{\mathrm{Co}, \mathrm{Cl}}$ : Eucinostomus currani y Eucinostomus melanopterus, Gerreidae. -del Atlántico Te, Ge: Eucinostomus melanopterus, Gerreidae.

-del Pacífico Te, Ge: Eucinostomus currani, Gerreidae.

Pez diablo Co, Fi. Hemiancistrus aspidolepis y Fonchiiichthys uracanthus, Loricariidae.

Pez gato ${ }^{\mathrm{Co}}, \mathrm{Fi}$ : Rhamdia guatemalensis, R. nicaraguensis, R. laticauda, Imparfinis lineatus y Pimelodella chagresi, Heptapteridae, Sciaedes guatemalensis, S. seemanni, Cathorops steindachneri y C. tuyra, Ariidae.

-acorazado Co, Fi: Fonchiiichthys uracanthus, Loricariidae.

-lápiz ${ }^{\mathrm{Co}, \mathrm{Fi}}$ : Trichomycterus striatus, Trichomycteridae.

Pez perro Co, Fi: Hoplias microlepis, Erythrinidae, Dormitator latifrons, D. maculatus, Eleotris amblyopsis, E. picta, E. pisonis y E. tecta, Eleotridae.

Pez plano Co, Fi. Citharicthys spilopterus, C. uhleri, Paralichthyidae.

Pez tigre ${ }^{\mathrm{Co}}$, Fi: Hoplias microlepis, Erythrinidae.

Picaculo $\mathrm{Co}, \mathrm{Cm}$ : Astyanax aeneus, Characidae, y Amatitlania kanna, Cichlidae.

Picalón Co, Cm: Pimelodella chagresi, Heptapteridae.

Picha/Pinga de/e padre Co, Fi. Rhamdia guatemalensis, R. laticauda y R. nicaraguensis, Heptapteridae.

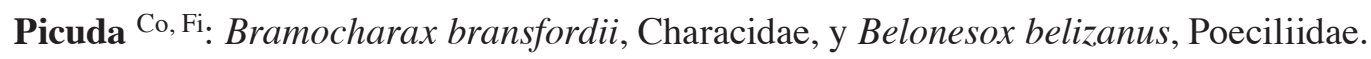

Piña sietecueros Co, Fi: Oligoplites saurus, Carangidae.

Pinto ${ }^{\mathrm{Co}}, \mathrm{Cl}$ : Parachromis managuensis (Günther, 1867), Cichlidae.

Pipa (Pez) ${ }^{\mathrm{C}}$, Fi. miembros de la familia Syngnathidae: Microphis lineatus, Pseudophallus elcapitanensis, P. mindii y P. starksi.

-de cola corta ${ }^{\mathrm{Te}}$, Fi: Microphis lineatus, Syngnathidae.

Pipón Co, Fi: Dormitator maculatus, Eleotridae, Poecilia gillii y P. mexicana, Poeciliidae.

Pís Pís Co: Vieja maculicauda, Cichlidae.

Plateada Co, $\mathrm{Cl}$ : Astyanax aeneus, A. coccibolca, Bramocharax bransfordii, Characidae, Atherinella argentea, A. chagresi, A. hubbsi, A. milleri, A. sardina, Atherinopsidae, y Cyphocharax magdalenae, Curimatidae.

Plateadita Co, $\mathrm{Cl}$ : Atherinella argentea, A. chagresi, A. hubbsi, A. milleri, A. sardina, Atherinopsidae, y Cyphocharax magdalenae, Curimatidae.

Pleco Co: Hemiancistrus aspidolepis y Fonchiiichthys uracanthus, Loricariidae.

Plecóstomo Co: Hemiancistrus aspidolepis y Fonchiiichthys uracanthus, Loricariidae; Plecóstomo es una palabra de origen griego, derivado de los vocablos "plekein" que significa plegar, trenzar y "stoma" que significa boca; Plecóstomo significa boca plegada.

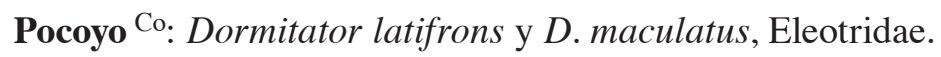

Robalito ${ }^{\mathrm{Co}}$, Fi: Centropomus robalito, Centropomidae. 
Róbalo/Robalo Co: miembros de la familia Centropomidae: Centropomus medius, $C$. nigrescens, $C$. parallelus, $C$. pectinatus, $C$. robalito, $C$. undecimalis, y $C$. viridis. -aleta amarilla $\mathrm{Co}, \mathrm{Cl}$ : Centropomus robalito, Centropomidae. -aleta manchada ${ }^{\mathrm{Co}}, \mathrm{Cl}$ : Centropomus medius, Centropomidae. -blanco ${ }^{\mathrm{Co}, \mathrm{Cl}}$ : Centropomus undecimalis, Centropomidae. -constantino ${ }^{\mathrm{Co}}$ : Centropomus pectinatus, Centropomidae. -escama pequeña ${ }^{\mathrm{Co}}$, Fi: Centropomus parallelus, Centropomidae. -negro ${ }^{\mathrm{Co}}, \mathrm{Cl}$ : Centropomus nigrescens, Centropomidae. -ojón Co, Fi: Centropomus medius, Centropomidae. -plateado ${ }^{\mathrm{Co}, \mathrm{Cl}}$ : Centropomus viridis, Centropomidae. -redondo ${ }^{\mathrm{Co}}, \mathrm{Fi}$ : Centropomus nigrescens, Centropomidae.

Roncador $\mathrm{Co}, \mathrm{Cm}$ : Pomadasys bayanus y P. crocro, Haemulidae. -de río del Atlántico ${ }^{\mathrm{Te}}$, Ge: Pomadasys croco, Haemulidae. -de río del Pacífico Te, Ge: Pomadasys bayanus, Haemulidae.

Ronco ${ }^{\mathrm{Co}}, \mathrm{Cm}$ : Pomadasys bayanus y P. crocro, Haemulidae.

Sabalete Co, Fi: Astyanax aeneus, A. coccibolca, Bramocharax bransfordii, Brycon behreae, B. costaricensis, Characidae, y Dorosoma chavesi, Clupeidae.

-montañero ${ }^{\mathrm{Co}, \mathrm{Ha}}$ : Agonostomus monticola y Joturus pichardi, Mugilidae.

Sábalo ${ }^{\mathrm{Co}}$ : Megalops atlanticus, Megalopidae, Brycon behreae, B. costaricensis, Astyanax aeneus, A. coccibolca y Bramocharax bransfordii, Characidae.

-real ${ }^{\mathrm{Co}}, \mathrm{Fi}$ : Megalops atlanticus, Megalopidae.

Saltón ${ }^{\mathrm{Co}}, \mathrm{Cm}$ : miembros de la familias Rivulidae: Cynodonichthys fuscolineatus, C. glaucus, C. hildebrandi, C. isthmensis, C. rubripunctatus, C. siegfriedi y C. uroflammeus.

Sardina Co: miembros de la familias Characidae: Astyanax aeneus, A. coccibolca, A. orthodus, Bramocharax bransfordii, Carlana eigenmanni, Hyphessobrycon panamensis, $H$. savagei, $H$. tortuguerae, Odontostilbe dialeptura, Pseudocheirodon terrabae, Pterobrycon myrnae, Roeboides bouchellei, R. bussingi, R. ilseae, Clupeidae: Dorosoma chavesi, Curimatidae: Cyphocharax magdalenae, y Atherinopsidae: Atherinella argentea, A. chagresi, A. hubbsi, A. milleri, A. sardina.

-blanca ${ }^{\mathrm{Co}}, \mathrm{Cl}$ : Astyanax orthodus, Characidae.

-común Co, Ha: Astyanax aeneus, Characidae.

-de río Co, Ha: Astyanax aeneus, Characidae, y Dorosoma chavesi, Clupeidae. -de vidrio ${ }^{\mathrm{Co}}, \mathrm{Fi} / \mathrm{Cl}$ : Roeboides bouchellei, R. bussingi y R. ilseae, Characidae.

-del lago Cocibolca Te, Ge: Astyanax cocibolca, Characidae.

-dientona ${ }^{\mathrm{Co}}$, Fi: Bramocharax bransfordii, Characidae.

-hocicuda ${ }^{\mathrm{Co}}$, Fi: Bramocharax bransfordii, Characidae.

-lagunera ${ }^{\mathrm{Co}, \mathrm{Ha}}$ : Astyanax cocibolca, Characidae. 
-picuda ${ }^{\mathrm{Co}}$, Fi: Bramocharax bransfordii, Characidae.

-plateada $\mathrm{Co}, \mathrm{Cl}$ : miembros de la familia Atherinopsidae: Atherinella argentea, A. chagresi, A. hubbsi, A. milleri y A. sardina.

Sardinilla Co, Fi: miembros de las familias Characidae: Astyanax aeneus, A. cocibolca, Roeboides bouchellei, R. bussingi, R. ilseae, Clupeidae: Dorosoma chavesi y Atherinopsidae: Atherinella argentea, A. chagresi, A. hubbsi, A. milleri y A. sardina.

Sardinita Co, Fi: miembros de la familia Characidae: A. cocibolca, Carlana eigenmanni, Hyphessobrycon panamensis, H. savage, H. tortuguerae, Odontostilbe dialeptura, Pseudocheirodon terrabae y Pterobrycon myrnae.

-de Myrna Te, Pe: Pterobrycon myrnae, Characidae.

-del Térraba Te, Ge: Pseudocheirodon terrabae, Characidae.

Sargentillo Co, $\mathrm{Cm}$ : Caranx caninus, Carangidae.

Sargento Co, Cm: Amatitlania kanna y A. siquia, Cichlidae.

Sierra (Pez) ${ }^{\mathrm{Co}}$, Fi: Pristis pristis, Pristidae.

Suela ${ }^{\mathrm{Co}, \mathrm{Fi}}$ : Achirus mazatlanus (PN, PS), Trinectes paulistanus y T. fonsecensis, Achiridae. -arepita ${ }^{\mathrm{Co}}, \mathrm{Fi}$ : Achirus mazatlanus; Achiridae.

-de cola manchada ${ }^{\mathrm{Te}, \mathrm{Cl}}$ : Trinectes fonsecensis; Achiridae.

-de Mazatlan Te, Ge: Achirus mazatlanus; Achiridae.

-rayada ${ }^{\mathrm{Co}}, \mathrm{Cl}$ : Trinectes fonsecensis; Achiridae.

Tarpón Co: Megalops atlanticus, Megalopidae, y Centropomus pectinatus, Centropomidae.

-del Atlántico Te, Ge: Megalops atlanticus, Megalopidae.

Tepemechín $\mathrm{Co}^{\mathrm{Cl}}$ : Agonostomus monticola, Mugilidae.

Tetra ${ }^{\mathrm{Co}}$ : miembros de la familia Characidae: Astyanax aeneus, A. cocibolca, A. orthodus, Bramocharax bransfordii, Carlana eigenmanni, Hyphessobrycon panamensis, $H$. savage, H. tortuguerae, Odontostilbe dialeptura, Pseudocheirodon terrabae y Pterobrycon myrnae.

-de Panamá, panameña Te, Ge: Hyphessobrycon panamensis, Characidae.

-de Savage Te, Pe: Hyphessobrycon savagei, Characidae.

-del Tortuguero ${ }^{\mathrm{Te}}, \mathrm{Ge}$ : Hyphessobrycon tortuguerae, Characidae.

-semaforo $\mathrm{Co}^{\mathrm{Cl}}$ : Pterobrycon myrnae, Characidae.

Tiburon toro ${ }^{\mathrm{Co}}$, Fi: Carcharhinus leucas, Carcharhinidae.

Tilapia ${ }^{\mathrm{Co}}$ : Oreochromis niloticus, Archocentrus multispinosus y A. centrarchus, Cichlidae. -del Nilo ${ }^{\mathrm{Te}}, \mathrm{Ge}$ : Oreochromis niloticus, Cichlidae.

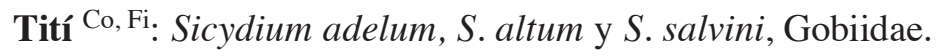

Trompetero ${ }^{\mathrm{Co}, \mathrm{Fi}}$ : miembros de la familia Syngnathidae: Microphis lineatus, Pseudophallus elcapitanensis, $P$. mindii y $P$. starksi.

Trucha Co: Oncorhynchus mykiss, Salmonidae.

-arcoiris ${ }^{\mathrm{Co}}, \mathrm{Cl}$ : Oncorhynchus mykiss, Salmonidae. 


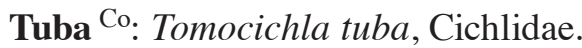

Vieja Co: Tomocichla tuba, T. sieboldii, Vieja maculicauda, Cichlidae, y Eleotris picta, Eleotridae.

Viejillo Co: Parachromis loisellei, Cichlidae.

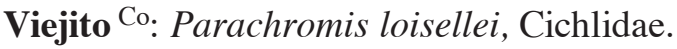

Viuda alegre ${ }^{\text {Co: }}$ Priapichthys panamensis, Poeciliidae.

Zapatero ${ }^{\mathrm{Co}, \mathrm{Fi}}$ : Fonchiiichthys uracanthus, Loricariidae.

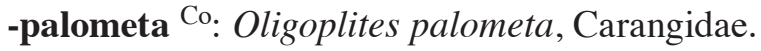

-sietecueros Co: Oligoplites saurus, Carangidae.

\section{Conclusiones}

Con este trabajo queda manifiesta la gran diversidad de nombres con los cuales se denomina comúnmente a las especies de peces dulceacuícolas de Costa Rica. Esta lista no es definitiva y será susceptible a corrección y ampliación en distintos aspectos; no obstante, se ofrece como base para futuros estudios.

El conocimiento de la biodiversidad constituye una de las bases necesarias para el manejo racional de los ecosistemas, fundamentalmente aquellos en los cuales habitan recursos explotados por el hombre. En los procesos actuales de conservación, donde se trata de involucrar de forma más activa a las comunidades, el conocer y poseer listados de nombres comunes de las especies, como el presente, permitirá una comunicación más fluida y una mayor y mejor integración entre personas comunes, técnicos, científicos y tomadores de decisiones, con miras a un objetivo común: la protección y el uso sostenible de los recursos naturales.

\section{Notas}

1. El autor agradece a Carlos Garita, Beatriz Naranjo, Bernald Pacheco, Carlos Sánchez, Andres Solano, Andres Arias, William Arias, Erika Solís, Daniela Ávila, Ana María Arias, Aldo Farah, Christian Trejos, Victor Cordero, Myrna López, William Bussing, Helena Molina, Gerardo Umaña, Monika Springer, Ana Rosa Ramírez, Rita Vargas, Prosanta Chakrabarty, Wilfredo Matamoros, Caleb McMahan, Joaquín Masís, Pedro Rocha, Clara Sibaja, Luis Arturo Angulo, Francisco Angulo, Sebastian Angulo y Carolina Méndez, entre muchas otras personas más, por toda su colaboración; desde brindar información relevante a los objetivos del trabajo, ayuda en la recolecta e identificación de especímenes, financiamiento parcial (cubriendo gastos de transporte, hospedaje y alimentación durante las salidas al campo a distintas localidades del país), hasta el espacio y las herramientas necesarias para la elaboración del manuscrito o apoyo para su culminación. Al Museo de Zoología, a la Escuela de Biología y al Centro de Investigación en Ciencias del Mar y Limnología (CIMAR) de la Universidad de Costa Rica, por el apoyo brindado a la presente investigación y al Sistema Nacional de Áreas de Conservación (SINAC) y al Ministerio de Ambiente y Energía (MINAE) quienes extendieron los respectivos permisos de investigación.

\section{Bibliografía}

Alpírez, Olivier. 1985. "Ictiofauna de la vertiente pacífica de Costa Rica”. Brenesia. 24: 297-318.

Angulo, Arturo, Carlos Alberto Garita-Alvarado, William Albert Bussing y Myrna Isabel López. 2013. "Annotated checklist of the freshwater fishes of continental and insular Costa Rica: additions and nomenclatural revisions”. Check List. 9 (5): 987-1019. 
Bussing, William Albert. 1998. Peces de las aguas continentales de Costa Rica/Freshwater fishes of Costa Rica. San José: Editorial Universidad de Costa Rica.

Bussing, William Albert y Myrna Isabel López. 1994. Peces demersales y pelagicos costeros del Pacífico de Centro América Meridional: guía ilustrada/Demersal and pelagic inshore fishes of the Pacific coast of lower Central America: an illustrated guide. San José: Editorial Universidad de Costa Rica.

2005. Peces de la Isla del Coco y peces arrecifales de la Costa Pacifica de Centro America Meridional/ Fishes of Cocos Island and reef fishes of the Pacific coast of lower Central America. San José: Editorial Universidad de Costa Rica.

2010. Peces costeros del Caribe de Centroamérica Meridional: guía ilustrada/Marine fishes of the Caribbean coast of lower Central America: an illustrated guide. San José: Editorial Universidad de Costa Rica.

Chebez, Juan Carlos, Alejandro Mouchard y Lucas Rodríguez. 2010. “Ornitonimia popular y científica de las aves argentinas. I. (Rheiformes)”. Nótulas faunísticas. 60: 1-13.

Eschmeyer, William. 2013. “Catalog of Fishes electronic version”. http://research.calacademy. org/ichthyology/catalog/fishcatsearch.html

Fournier, Luis y Elmer Guillermo García. 1998. Nombres vernaculares y científicos de los árboles de Costa Rica. San José: Guayacán.

León, Jorge y Luis Poveda. 2000. Nombres comunes de las plantas en Costa Rica. San José: Guayacán.

Martinez, Guillermo y Argentino Bonetto. 1957. "Nota acerca de los nombres vernaculos de peces en el Parana medio". Anales del Museo Provincial de Ciencias Naturales Florentino Ameghino. 2: 1-10.

Mones, Alvaro y Ursula Kuhl. 1991. "Comentarios sobre el uso de nombres vulgares en Zoología”. Anales Instituto de Biología Universidad Nacional Autónoma de México. 62: 249-252.

Mora, José. 2000. Mamíferos silvestres de Costa Rica. San José: Editorial Universidad Estatal a Distancia.

Navas, Jorge, Tito Narosky, Nelly Bó y Juan Carlos Chebez. 1991. Lista patrón de los nombres comunes de las Aves Argentinas. Buenos Aires: Asociación Ornitológica del Plata.

Pardos, Francisco. 2001. “¿Cómo llamar a los animales? Nombres vulgares y nombres científicos”.Panace@.2: 56-61.

Sandoval, Luis. 2006. "Nombres comunes de las aves de Costa Rica: significado y origen". Revista de Filología y Lingüística. 32 (1): 247-259.

Vuilleumier, François. 1999. "Sobre la necesidad de estandarizar los nombres en castellano y portugués de las aves neotropicales”. Ornitología Neotropical. 10: 69-75. 
